\title{
Inhibition of the IL-17A axis Protects against Immune-related Adverse Events while Supporting Checkpoint Inhibitor Anti-tumor Efficacy
}

Melissa Lechner ( $\square$ mlechner@mednet.ucla.edu )

UCLA Geffen School of Medicine https://orcid.org/0000-0002-4744-6566

Anushi Patel

UCLA Geffen School of Medicine

Willy Hugo

UCLA

Trevor Angell

USC Keck School of Medicine

Ho-Chung Chen

UCLA Geffen School of Medicine

Marissa Pioso

UCLA Geffen School of Medicine

Aline Hoang

UCLA Geffen School of Medicine

Natalie Yakobian

UCLA Geffen School of Medicine

Ethan McCarthy

UCLA Geffen School of Medicine

Eduardo Rodriguez

UCLA Geffen School of Medicine

Mandy Cheng

UCLA Geffen School of Medicine

Lily Guo

UCLA Geffen School of Medicine

Alexandra Drakaki

UCLA Geffen School of Medicine

Antoni Ribas

University of California, Los Angeles https://orcid.org/0000-0003-3669-8458

Marueen Su

University of North Carolina 


\section{Article}

Keywords: Checkpoint Inhibitor Immunotherapy, Immune Related Adverse Events, Thyroiditis, Type 3 Immune Cells

Posted Date: June 30th, 2021

DOl: https://doi.org/10.21203/rs.3.rs-609476/v1

License: (c) (i) This work is licensed under a Creative Commons Attribution 4.0 International License. Read Full License 
Inhibition of the IL-17A axis Protects against Immune-related Adverse Events while Supporting Checkpoint Inhibitor Anti-tumor Efficacy

Melissa G. Lechner, MD, $\mathrm{PhD}^{1^{*}}$, Anushi Y. Patel ${ }^{2}$, Willy Hugo, $\mathrm{PhD}^{3}$, Trevor E. Angell, MD ${ }^{4}$, HoChung Chen, BS², Marissa S. Pioso, BS ${ }^{2}$, Aline T. Hoang, BS ${ }^{2}$, Natalie Yakobian, BS ${ }^{2}$, Ethan C. McCarthy ${ }^{2}$, Eduardo D. Rodriguez, MD ${ }^{5}$, Mandy I. Cheng, BA ${ }^{2}$, Lily Guo, BS², Alexandra Drakaki, $\mathrm{MD}, \mathrm{PhD}^{6}$, Antoni Ribas, $\mathrm{MD}, \mathrm{PhD}^{6}$, and Maureen $\mathrm{A} . \mathrm{Su}, \mathrm{MD}^{2,7}$

\section{Affiliations:}

1. Division of Endocrinology, Diabetes, and Metabolism, UCLA David Geffen School of Medicine, Los Angeles, CA

2. Department of Microbiology, Immunology, and Molecular Genetics, UCLA David Geffen School of Medicine, Los Angeles, CA

3. Division of Endocrinology and Diabetes, USC Keck School of Medicine, Los Angeles, CA

4. Division of Dermatology, Department of Medicine, UCLA David Geffen School of Medicine, Los Angeles, CA

5. Department of Pathology, UCLA David Geffen School of Medicine, Los Angeles, CA

6. Division of Hematology and Oncology, UCLA David Geffen School of Medicine, Los Angeles, CA

7. Division of Pediatric Endocrinology, UCLA David Geffen School of Medicine, Los Angeles, CA 
Checkpoint inhibitor (ICI) immunotherapy leverages the body's own immune system to

27 attack cancer cells but leads to unwanted autoimmune side effects in up to $60 \%$ of patients.

28 Such immune related adverse events (IrAE) lead to treatment interruption, permanent 29 organ dysfunction, hospitalization and premature death. Thyroiditis is one of the most 30 common IrAE, but the cause of thyroid IrAE remains unknown. Here we present a novel 31 mouse model in which checkpoint inhibitor therapy leads to multi-organ autoimmune

32 infiltrates and show that activation and infiltration of Type 3 immune cells including IL17A ${ }^{+}$

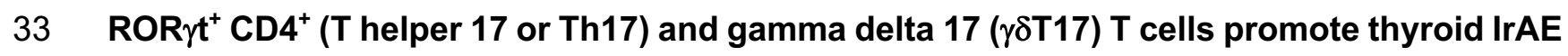

34 development. In parallel, Th17 and $\gamma \delta \mathrm{T} 17$ cells were similarly expanded in cancer patients

35 treated with ICI. Furthermore, antibody-based inhibition of IL-17A, a clinically available

36 therapy, significantly reduced thyroid IrAE development in ICI-treated mice. Finally,

37 combination of IL-17A neutralization with ICI treatment in a mouse tumor model did not

38 reduce $\mathrm{ICI}$ anti-tumor efficacy and indeed showed a trend toward enhancement. These

39 studies suggest that targeting Th17 and $\gamma \delta 17$ function may reduce IrAE without impairing

$40 \mathrm{ICI}$ anti-tumor efficacy and may be a generalizable strategy to address IL17-mediated IrAE. 


\section{INTRODUCTION}

42 Immune checkpoint inhibitors (ICl) against programmed death protein (PD-1) and cytotoxic T 43 lymphocyte antigen (CTLA)-4 have revolutionized cancer therapy and hold great promise for the

44 treatment of advanced malignancies ${ }^{1}$. Since initial approval in 2011, seven ICI have entered 45 clinical care, with nearly half of patients with cancer in the U.S. eligible for $\mathrm{ICI}$ treatment ${ }^{2}$. ICI are 46 used for first line therapy of metastatic renal, bladder, head and neck, liver, and certain lung, 47 colon, and breast cancers ${ }^{2}$. However, the benefits and use of $\mathrm{ICI}$ are limited by development of 48 autoimmune side effects, termed immune related adverse events (IrAEs) seen in up to $60 \%$ of 49 patients $^{3-5}$. Endocrine, gastrointestinal and liver, and dermatologic tissues are some of the most 50 common affected by IrAEs ${ }^{3,4,6}$. Pulmonary, neurologic, renal, and cardiac autoimmune side effects

51 from $\mathrm{ICl}$ are less frequent but associated with grave clinical consequences, including mortality

52 rates of $17-40 \%{ }^{7}$. IrAEs can lead to permanent organ dysfunction, cancer therapy interruption,

53 hospitalization, and premature death ${ }^{5,7}$. With the expanding use of ICls, IrAEs represent a growing 54 clinical problem.

56 Despite significant efforts to date, the mechanisms of IrAE remain poorly understood ${ }^{8}$. Thyroiditis 57 is one of the most common ICl-associated IrAE, occurring in approximately $10-15 \%$ of patients 58 treated with anti-PD1/L1 monotherapy and 30\% of patients treated with combination anti-PD-1/L1 59 and CTLA-4, $4^{6,9,10}$. Like IrAE in other organs, ICl-thyroiditis has both overlapping and distinct 60 features with spontaneous autoimmunity (i.e. Hashimoto's thyroiditis, HT) ${ }^{9-12}$. Two prior important 61 studies ${ }^{13,14}$ showed lymphocyte accumulation in ICI-thyroiditis, similar to $\mathrm{HT}$. The onset of 62 thyroiditis during ICl-treatment may be due to the release of immune checkpoints on pre-existing, 63 thyroid-reactive T cells, as suggested by the progressive loss of gland function in patients with $64 \mathrm{HT}$ and increased risk of ICl-thyroiditis in patients with baseline thyroid autoantibodies to thyroid 65 peroxidase $(\mathrm{TPO})^{13,15,16}$. On the other hand, thyroid gland destruction in ICI-thyroiditis is much 66 more rapid compared to spontaneous HT and usually with a clear hyperthyroid phase during gland 
67 destruction and subsequent evolution to permanent hypothyroidism ${ }^{9,12}$. Many patients with $\mathrm{ICl}-$

68 thyroiditis also lack the usual anti-thyroid antibodies to TPO or thyroglobulin (Tg) seen in HT at 69 diagnosis ${ }^{9,12}$. Thus $\mathrm{ICl}$-thyroiditis, while similar to $\mathrm{HT}$, may be driven by the additional presence 70 of novel immune mechanisms and antigens.

72 To develop ICI therapies with less toxicity while preserving anti-tumor efficacy, we must determine

73 the cause of IrAE. Currently guidelines recommend suspension immunotherapy and high dose

74 glucocorticoid therapy for severe manifestations (grade 3 or 4 ) of most IrAE ${ }^{17,18}$, which may 75 unnecessarily impair the efficacy of $\mathrm{ICl}$ cancer treatment for many patients ${ }^{5,19}$. It is now recognized

76 that high dose glucocorticoid therapy does not attenuate or prevent thyroiditis, hypophysitis, or 77 diabetes, and correlates with shorter overall survival ${ }^{12,20,21}$. Recent data in gut ICl-induced IrAE 78 suggested that immune adverse events may be uncoupled from anti-cancer efficacy using anti79 inflammatory treatments ${ }^{22}$. Studies of ICl-associated IrAE in the thyroid may identify common

80 mechanisms driving IrAE development in organs with more grave clinical consequences (e.g.

81 pneumonitis). In this study, we define immune mechanisms underlying thyroid IrAE from

82 checkpoint immunotherapy using a newly developed mouse model and translational studies in

83 patients with $\mathrm{ICl}$-thyroiditis. High dimensional analysis of peripheral and thyroid-infiltrating

84 immune cells revealed recruitment and activation of type 3 immune cells including IL $17 A^{+} \mathrm{ROR}_{\gamma \mathrm{t}^{+}}$

$85 \mathrm{CD}^{+}$(T helper 17 or Th17) and gamma delta $17(\gamma \delta \mathrm{T} 17) \mathrm{T}$ cells. Moreover, pre-clinical studies

86 demonstrate that IL-17 blockade effectively protects against ICl-thyroiditis without negatively

87 impacting ICl-mediated anti-tumor immunity.

88

89 RESULTS

90 The NOD background strain predisposes mice to ICI-IRAEs that mimic those seen in humans 
91 The study of ICl-associated IrAE has been hindered by the lack of robust preclinical models ${ }^{8}$.

92 While prior studies have reported autoimmunity in ICl-treated mice, these models on the C57BL/6

93 (B6) background developed minimal ICI-IrAEs. Augmenting these mild phenotypes required

94 significant manipulation, such as regulatory $\mathrm{T}$ cell depletion ${ }^{23}$, genetic knock-in of human 95 checkpoint proteins ${ }^{24}$, chemical stimulants ${ }^{25}$ or co-administration of complete Freund's adjuvant ${ }^{19}$.

96 A recent paper utilized CBA/J mice in a thyroid IrAE model, but IrAE induction required non-

97 physiologic immunization with human thyroglobulin ${ }^{26}$. We sought to develop a murine model of

98 ICI-IrAE that better recapitulated the development of IrAE in patients; namely, treatment-

99 dependent incident multi-system autoimmune infiltration in an immune competent host. We first

100 sought to establish a baseline for ICI-IrAE in the wild type B6 strain. Groups of four- to six-week

101 old B6 mice were treated twice weekly with anti-mouse CTLA-4 (clone 9D9) and PD-1 (RPM1-

102 14) antibodies or isotype controls (2A3, MPC-11), at 10 mg/kg/dose i.p., as outlined in Fig.1a. We

103 chose to treat with dual ICI therapy (combination anti-CTLA-4/PD-1) since the combination has

104 been linked with the highest rates of ICI-IrAEs ${ }^{3,4}$. Mice were monitored at least twice weekly for

105 signs of autoimmunity, including weight loss, decreased activity, and glucosuria. After 4 weeks of

106 treatment, mice were sacrificed, and multiple tissues (salivary, lacrimal, pancreas, liver, lung,

107 heart, colon, eye, gonad, and thyroid) were evaluated by histology and flow cytometry for the

108 development of autoimmune infiltrates. Tissue immune infiltration was quantified by blinded

109 assessment of hematoxylin and eosin (H\&E)-stained formalin fixed paraffin-embedded (FFPE)

110 sections (5 high powered fields/section in each animal). Sera were collected for measurement of

111 thyroid autoantibodies to $\mathrm{Tg}$ and TPO, as previously described ${ }^{27}$. Overall mice were resistant to

$112 \mathrm{ICl}$-induced autoimmunity with immune infiltration limited on average to two organs per mouse

113 (Fig. 1b). Increased immune infiltrates were seen in the liver and lacrimal glands, but not in the

114 thyroid (Fig. 1b and Extended Data Fig. 1). Additionally, B6 mice did not develop thyroid 115 autoantibodies to thyroid peroxidase (TPO) or thyroglobulin (Tg) (Fig. 1c). Furthermore, weights

116 remained stable in $\mathrm{ICl}$ and isotype treated mice, and survival was unchanged by $\mathrm{ICI}$ treatment 
117 during the experimental period (data not shown). Similar results were seen in mice treated with

$118 \mathrm{ICl}$ or isotype for eight weeks (data not shown). In summary, B6 mice failed to develop significant 119 multisystem autoimmunity with combination anti-PD1 and anti-CTLA-4 immune checkpoint 120 inhibitor (Dual $\mathrm{ICl}$ ) therapy. These findings are in keeping with previous reports that B6 mice are 121 resistant to spontaneous autoimmunity, perhaps due to genetic polymorphisms that alter immune 122 responses in this inbred strain $^{28,29}$.

124 Underlying subclinical autoimmunity or genetic predisposition increases risk of developing IrAE 125 during $\mathrm{ICl}$ cancer therapy in patients ${ }^{15,20,30}$. For example, patients with thyroid auto-antibodies 126 against thyroid peroxidase at baseline were five-fold more likely to develop thyroiditis with $\mathrm{ICI}$ 127 treatment ${ }^{11,31}$. The NOD mouse is an autoimmune-prone inbred strain that is best known for its contributions to type 1 diabetes studies but has also been invaluable in understanding generalized autoimmunity $^{32}$. NOD mice develop spontaneous autoimmunity in multiple tissues including 130 thyroiditis, at low frequency $(14 \% \text { at } 52 \text { weeks })^{33,34}$. Thus, we tested whether IrAE development 131 may be more robust and reproducible in NOD mice compared to B6. As done in B6 mice, four- to 132 six-week old NOD mice were treated twice weekly with anti-mouse CTLA-4, PD-1, both (Dual ICI), 133 or isotype control and then autoimmune tissue infiltrates and thyroid autoantibodies evaluated.

135 ICl-treated NOD mice developed increased immune infiltrates in multiple tissues (e.g. thyroid, 136 colon, liver, lung, salivary and lacrimal glands) compared to isotype-treated animals, as shown in 137 Fig. 1d-e and Extended Data Fig. 2 (ANOVA p<0.0001; $p$ values vs. isotype were $<0.0001$ for 138 anti-CTLA-4, anti-PD-1, and Dual ICI). The predominant inflammation in the thyroid consisted of 139 lymphocytic aggregates within the interstitium and occasionally perivascular aggregates. In the 140 liver, both focal collections of lymphocytes within liver parenchyma and peri-portal venule sites 141 were seen. Lung tissues similarly showed aggregates of focal lymphocyte aggregation, as well 142 as loss of alveolar air spaces. Histologic examination of the colon sections showed areas with 
143 neutrophilic cryptitis, crypt epithelial apoptosis, and rare neutrophilic microabscesses. Increased

144 intraepithelial lymphocytes were not detected, but basal lymphoplasmacytosis was focally seen.

145 The pattern of immune infiltration in the kidney was primarily glomerulonephritis with accumulation

146 of lymphocytes, and occasionally peri-vascular lymphocytic aggregates. In the pancreas, immune

147 infiltration was centered on islet and peri-vascular areas, with lymphocytic infiltration of $25-100 \%$

148 of islets in most ICl-treated animals. Finally, salivary and lacrimal glands showed interstitial

149 lymphocyte aggregates often with diffuse gland involvement. One animal developed pericardial

150 inflammation. Eye, adrenal, myocardial, and gonad tissues were also evaluated, and autoimmune

151 infiltrates were not seen in any animals. As in patients, autoimmunity occurred more frequently

152 with the combination of anti-PD-1 + anti-CTLA-4 vs. with single agent ICI. In addition, mice

153 developed thyroid autoantibodies to TPO and Tg (Fig. 1f) and, as expected, showed acceleration

154 of underlying autoimmune risk (i.e. diabetes, data not shown) $)^{11,20}$. Mice developing diabetes were

155 treated with insulin, as described previously ${ }^{35}$. Thyroid hormone status was evaluated at 8 weeks

156 by serum measurement of free thyroxine. ICl-treated mice had increased mean thyroxine serum

157 concentrations compared to isotype controls, consistent with excess thyroid hormone release and

158 hyperthyroxinemia during the early destructive phase of ICl-thyroiditis (Fig. 1g). Furthermore, 159 some ICl-treated mice notably exhibited low serum thyroxine consistent with the conversion to 160 hypothyroidism over time in ICl-thyroiditis and mirroring the earlier conversion to hypothyroidism 161 in Dual vs. single agent therapy seen in patients. ${ }^{9}$

163 During the 8-week treatment period, the rate of spontaneous autoimmunity was low in isotype164 treated controls which developed only pancreatic insulitis and occasional lacrimal or salivary 165 gland focal immune infiltration at 12-15 weeks of age (Fig. 1d-e), consistent with their NOD 166 background. Thus, our mouse model recapitulates critical features of clinical ICl-associated 167 autoimmunity and thus will facilitate studies of the mechanisms leading to IrAEs. 
170 To define immune changes occurring with $\mathrm{ICl}$-associated autoimmunity, we measured the

171 frequency and activation status of different immune populations in ICl- vs. isotype-treated mice.

172 In the ICl-treated animals, we noted increased frequency of pan-CD3 ${ }^{+} \mathrm{T}$ cells among splenocytes

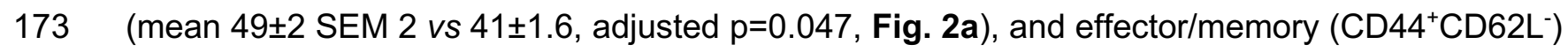

174 subsets among $\mathrm{CD}^{+}$and $\mathrm{CD}^{+} \mathrm{T}$ cells, compared to isotype antibody-treated control mice (Fig.

$175 \mathbf{2 b}$; adjusted $p<0.001$ for both). No difference (or a slight decrease) was seen in the frequency of

176 B cells $\left(B 220^{+}\right.$, mean $41 \pm 1.8$ vs $\left.46.6 \pm 1.6, p=n s\right)$, dendritic cells (CD11 $c^{+} C D 11 b^{\text {low }}$, mean $1.4 \pm 0.1$

177 vs 1.7 $\pm 0.1, p=n s)$ and myeloid cells (CD11 ${ }^{+} C D 11 c^{-} B 220^{-}$, mean $2.5 \pm 0.2$ vs 2.8 $\left.\pm 0.2, p=n s\right), N K$

178 (NKp46 ${ }^{+} \mathrm{CD}^{-}$, mean $1.5 \pm 0.1$ vs $\left.2.8 \pm 0.2, \mathrm{p}<0.0001\right)$ and NKT cells $\left(\mathrm{NKp} 46^{+} \mathrm{CD}^{+}\right.$, mean $0.3 \pm 0.02$

179 vs. $0.5 \pm 0.01, p<0.0001$ ) (Extended Data Fig. 3). T cell expansion and activation following anti-

180 PD-1 and/or anti-CTLA-4 treatment has been reported previously ${ }^{36,37}$. We then evaluated cytokine

181 production by $\mathrm{T}$ cells in Dual ICl-treated mice to understand how their activation may skew overall

182 immune responses and potentially lead to the observed autoimmunity. We saw no significant

183 difference in interferon (IFN)- $\gamma$ producing $C D 4^{+} \mathrm{T}$ cells from ICl-treated $v s$. isotype mice $(2.1 \pm 0.3$

184 vs. $2.6 \pm 0.4$ percent of $\mathrm{CD} 4+\mathrm{T}$ cells, $\mathrm{p}=\mathrm{ns}$, Fig. $2 \mathrm{c}$ ). IL-17A $\mathrm{I}^{+} \mathrm{CD}^{+}$helper $\mathrm{T}$ cells (Th17), on the 185 other hand, were significantly increased in ICl-treated mice compared to isotype controls $(2.1 \pm 0.1$

186 vs. $1.4 \pm 0.2$ percent of $\mathrm{CD}^{+} \mathrm{T}$ cells, $\mathrm{p}<0.001$, Fig. $2 \mathrm{~d}$ ). Consistent with this finding, $\mathrm{CD} 4^{+} \mathrm{T}$ cells

187 in ICl-treated mice also had increased expression of Th17-associated transcription factor ROR $\gamma \mathrm{t}$

188 (Fig. 2e). IL-17A-expressing $\mathrm{CD}^{+}$Th17 cells have been previously implicated in the 189 pathogenesis of multiple spontaneous autoimmune diseases, including Hashimoto's thyroiditis, 190 but have yet to be reported in $\operatorname{IrAE}^{38-41}$. Surprisingly, IL-17A ${ }^{+}$immune cells lacking CD4 191 expression (CD4-) were also significantly increased in ICl-treated mice (Fig. 2f, p=0.005). IL-17A 192 can be produced not only by Th17 but also by other immune cells including IL-17-producing 193 gamma delta cells ( $\gamma \delta$ T17) and Group 3 innate lymphoid cells (ILC3s) $)^{42-44}$. Thus it is possible that 
194 these CD4- $\mathrm{IL}^{-17 \mathrm{~A}^{+}}$cells represent gamma delta T cells or innate lymphoid cells. As shown in

195 Fig. 2g, IL-17A $\gamma \delta$ T cells were indeed increased in ICl-treated compared to isotype mice 196 ( $p=0.03)$. From these data, we hypothesized that IL-17A-producing cells from multiple lineages, 197 including Th17 and $\gamma \delta \mathrm{T} 17$, contribute to ICl-immune-related toxicities in our mouse model.

\section{Diverse infiltrating immune cells in the thyroid of ICl-treated mice}

200 Given that ICI treatment increases thyroid immune infiltration in NOD mice (Fig. 1), we used a 201 multi-modal strategy to delineate the cellular composition within the thyroid infiltrate. 202 Immunohistochemical staining for canonical immune markers on FFPE sections showed the 203 presence of $T$ cells (CD3), macrophages (F4/80), and B cells (B220) in immune aggregates in 204 ICl-treated mice, with rare immune cells in the thyroid parenchyma of isotype controls (Fig. 3a). 205 We then quantified immune cells in thyroid tissues using flow cytometry as shown in Fig. 3b-c. 206 Fresh thyroid tissues from $\mathrm{ICl}$ or isotype-treated mice were perfused with saline and dissociated 207 into single cell suspensions then stained for immune markers and analyzed for CD45 ${ }^{+}$-gated 208 immune cells. More infiltrating CD45 ${ }^{+}$immune cells were seen in thyroid tissue of ICl-treated mice 209 compared to isotype controls (mean 15,875 cells/thyroid \pm SEM 7,515 vs. 1,195 \pm 687 , trend, $210 \mathrm{p}=0.057)$ (Fig. 3b). T cells $\left(\mathrm{CD}^{+}\right)$constituted the major group of the infiltrating immune cells $211\left(\mathrm{CD}^{2} 5^{+}\right)$in thyroid tissue and were significantly increased in ICl-treated mice (mean 3,332 $\pm 1,220$ 212 cells/thyroid lobe vs. $420 \pm 176, p=0.02$ ). Other populations seen in thyroid immune infiltrates were 213 also more numerous in ICl-treated mice, including DCs (CD11 ${ }^{+}$CD3- F4/80- B220- CD11 b ${ }^{\text {low }}$,

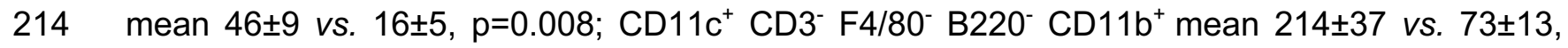
$215 \mathrm{p}=0.001)$, macrophages $\left(\mathrm{F} 4 / 80^{+}\right.$, mean $234 \pm 43$ vs. $\left.140 \pm 25\right)$, B cells $\left(B 220^{+} \mathrm{CD}^{-} \mathrm{CD}^{-11 \mathrm{~b}^{-} \text {, mean }}\right.$ $216212 \pm 77$ vs. $32 \pm 13, p=0.03$ ), and NK cells (NKp46 ${ }^{+}$CD3-, mean $221 \pm 74$ vs. 46 $\pm 16, p=0.03$ ) (Fig. 217 3c). This diversity of immune cell populations suggests the dynamic interplay of multiple immune 218 cell types in the autoimmune response. 
220 With the goal of precisely understanding the cellular composition and cell type-specific 221 inflammatory networks associated with thyroid IrAEs at a single cell resolution, we turned to single 222 cell RNA sequencing (scRNAseq) of CD45 thyroid-infiltrating immune cells from Dual ICl-treated 223 mice (Fig. 3d). This technique facilitates identification of rare cell populations and characterization

224 of limited tissue specimens, as in the case of murine thyroid tissue (approximately $1 \mathrm{~mm}^{3}$ of thyroid 225 tissue per animal). Sorted, live $C D 45^{+}$cells from Dual ICl-treated NOD mice thyroid specimens 226 ( $n=10$ animals) were used to construct Chromium ${ }^{\mathrm{TM}} 10 \mathrm{x}$ single cell $5^{\prime}$ gene expression libraries 227 for single cell sequencing. The reads were mapped to the mouse reference genome using the 228 CellRanger pipeline. The transcript counts of each cell were subsequently analyzed using the 229 Seurat R package ${ }^{45}$. Briefly, the cells from each sample were initially QC-ed to remove artifacts 230 with low sequencing coverage and/or high mitochondrial reads. The cells passing the QC were 231 clustered in order identify different immune cell populations. Fourteen heterogeneous immune 232 cell populations were identified and visualized using the uniform manifold approximation and 233 projection (UMAP) (Fig. 3d; total $n=4,095$ cells). Putative cell cluster identities in Fig. 3d were 234 made based upon expression of canonical population markers and functional gene expression 235 (Extended Data Fig. 4a-b and Extended Data Table 1). Specifically, lymphoid cell clusters 236 consisted of $\mathrm{CD}^{+} \mathrm{T}$ cells (Cd4: Cd3e, Cd4), CD8 ${ }^{+} \mathrm{T}$ cells (Cd8: $\left.\mathrm{Cd} 3, \mathrm{Cd} 8 \mathrm{a}\right)$, and gamma delta $237(\gamma \delta)$ T cells $(C d 3 e, T r d c)$. T cell clusters were further defined as naive CD4 (Sell, Ccr7, Satb1, 238 Foxp1, Lef1, S1pr1), regulatory T cells (Treg: Cd3e, Cd4, Foxp3, Ctla4, Ikzf2, Tnfrsf18 (GITR), 239 ICOS), effector CD4 (Cd4, Cd40lg, Sh3bgrl3, Cd52, Id2, Ly6a, Cxcr3, Tmem176a/b; or Cd4, 240 Itga4, Eef1a1, Cd40lg, Cxcr3, Fasl, Icos, Ctla4, Id2, Nrp1; or Cd4, Ctla4, Cd40lg, Fasl, Cxcr4, 241 Ifng, Tnfrsf4, Tbx21, ICOS, Tnfaip3, Nfkbia), and effector CD8 (Cd8a, Cd8b1, Gzmk, Ccl5, Nkg7, 242 Ly6a, Gzma, KIrd1, Prf1). Myeloid cell populations included macrophages (Cd68, Cd14, Fcgr1, 243 Adgre1, C1qa, C1qb, C1qc, Csfr1, Cd74) and (monocytic) dendritic cells (DC/APC: Cd68, Cd74, 244 Cd14, Itgax, H2-Aa). Other immune clusters were innate lymphoid cells (ILC: II7r, I/1rb, I/13, Arg1), 
natural killer T cells (NK/NKT: Ncr1, Cd7, Klra5, Klrd1, Fcer1g, Gzma), and B cells (B: Cd79a, Cd19, Ebf1, Cd74). These data confirm and provide further characterization of the diverse thyroid-

247 infiltrating immune cell populations seen by IHC and flow cytometry techniques in ICl-treated NOD 248 mice.

Infiltrated thyroids of ICl-treated mice accumulate multiple lineages of Rorc-expressing 251 immune cells

252 Single-cell RNA sequencing, as well as flow cytometry, IHC, and qRT-PCR, studies showed 253 marked T cell thyroid infiltration with ICI treatment (Fig. 3a, c, and e). Therefore, we sub-clustered $254 \mathrm{Cd} 3 \mathrm{e}^{+}$cells to further delineate the T cell populations present and their functional status (Fig. 3f, $255 n=3,178$ cells). This identified 10 populations including $\mathrm{CD}^{+}$(clusters $\left.0,1,4,7\right), \mathrm{CD}^{+}(2)$, mixed $256 \mathrm{CD}^{+}$and $8^{+}(8,9)$ and $\operatorname{TCR}_{\gamma} \delta^{+}(3,5,6)$ subsets (Fig. 3f, Extended Data Fig. 4c, and Extended 257 Data Table 1). Thyroid tissue infiltrating $\mathrm{T}$ cells showed gene expression associated with 258 activation (Cd44, Cd69) and effector functions, including type 1 (Tbx21) and type 3 (Rorc) 259 immunity and cytotoxicity (T cell subcluster 2: Cd8a, Cd8b, Gzmk, Cc/5, Fasl, Prf1, KIrd1, KIrc1, 260 Gzmk, Ly6a, Eomes; and subcluster 6: Ncr1, Trdc, KIra5, KIra4, KIra1, KIrd1, Cd7, Nkg7). Type 2611 immunity and cytotoxic T cells have previously been reported in ICI treatment and are thought 262 to be important for anti-tumor effects ${ }^{36,46}$. However, a role for Type 3 immune responses in ICI 263 treatment is less clear.

265 RORc expression, associated with Type 3 immunity, was highest in subclusters 3 and 5 (Fig. 3g). 266 Both Rorc ${ }^{+}$clusters also coordinately expressed Trdc, Tcrg-V6, Rora, Tmem1276b, Tmem176a, 267 Cxcr6, and Serpinb1a, supporting their inclusion of $\gamma \delta T 17$ cells (Extended Data Fig. 4c and 268 Extended Data Table 1) ${ }^{47}$. We confirmed increased expression of Rorc and associated 269 Th17/ $\gamma \delta$ T17 pathway genes //23r and //17a using qRT-PCR. As shown in Fig. 3h, expression was 
270 significantly increased in ICl-treated vs. isotype mice for Rorc [mean fold change (FC) $2.4 \pm 0.1$, $271 \mathrm{p}<0.001]$, II23r (FC 1.28 $\pm 0.09, \mathrm{p}<0.001)$, and II17a (FC 1.45 $\pm 0.07, \mathrm{p}<0.001)]$. In addition, flow

272 cytometry analysis of infiltrating immune cells dissociated from fresh thyroid showed increased

273 intrathyroidal ROR $\gamma \mathrm{t}^{+}$pan-CD3 ${ }^{+} \mathrm{T}$ cells, which include Th17 and $\gamma \delta \mathrm{T} 17$ cells, in Dual ICl-treated

274 mice compared to isotype controls (mean $936 \pm 267$ vs. $183 \pm 43$ cells per thyroid lobe, Fig. $3 \mathbf{3}$ ).

275 These data, together with our earlier data that Th17 and $\gamma \delta \mathrm{T} 17$ cells were increased in the spleen 276 of ICl-treated mice (Fig. 2), suggest a potential role for ROR $\gamma \mathrm{t}^{+}$Th17 and/or $\gamma \delta \mathrm{T} 17$ cells in the 277 development of ICl-associated thyroiditis.

279 To better characterize the $\mathrm{RORc}^{+}$populations in thyroid immune infiltrates, we sub-clustered $\mathrm{T}$ 280 cells with detectable RORc expression (Rorc > 1) (Fig. 4a and Extended Data Fig. 4e). This 281 analysis produced 3 subclusters $(0,1,2)$ : Subclusters 0,1 , and 2 expressed high levels of $\gamma \delta \mathrm{T}$ cell 282 markers ( $T r d c, T r g-V 6)$, and Subcluster 2 also included $C D 4^{+}$T helper cells (Cd4). Subclusters 0 283 and 1 differentially expressed $/ / 23 r$ (Fig. 4b), suggesting two $\gamma \delta \mathrm{T} 17$ cell states that could be 284 distinguished by II23r. Moreover, Pdcd1 was expressed by all three subclusters and Ctla4 was 285 highly expressed by Subcluster 2, which suggests that all three subclusters can be activated by 286 anti-PD1 and/or anti-CTLA-4 checkpoint inhibitors (Fig. 4c). We also queried the expression of 287 cytokine receptors previously reported to promote induction of $\gamma \delta \mathrm{T} 17$ cells, namely Tnfrsf1a and $288 \| 1 / 11^{42}$. Subclusters 0 and 1 expressed the highest levels of Tnfrsf1a, while Subclusters 0,1 , and 2892 each expressed I/1r1 (Fig. 4d). Thus, cytokine receptors needed for $\gamma \delta \mathrm{T} 17$ differentiation are 290 indeed present in these Rorc ${ }^{+}$T cells. In support of an important role for TNF $\alpha$ and IL-1 $\beta$ in $\gamma \delta \mathrm{T} 17$ 291 cell induction, intrathyroidal myeloid cells strongly expressed inflammatory cytokine genes Tnf 292 and II1b (Extended Data Fig. 5a-c) in scRNAseq data. Increased intrathyroidal TNF $\alpha$ and IL-1 $\beta$ 293 was confirmed using qRT-PCR comparing ICl-treated to isotype mice (Extended Data Fig. 5d). 294 Of note, TNF $\alpha$ and IL-1 $\beta$ also amplifies Th17 differentiation ${ }^{48}$, and additional cytokines associated 
295 with Th17 development (TGF $\beta$ and IL-6) were also increased, although to a lesser degree, in

296 thyroids of ICl-treated mice (Extended Data Fig. 5c-d) ${ }^{38}$. Finally, predicted ligand-receptor 297 interactions in scRNAseq data showed significant cell-cell communication from myeloid 298 populations to T cells via CXCL and CCL family chemokines (Extended Data Fig. 5e and 299 Extended Data Table 2). Interestingly, CXCL communication arose singularly from the predicted 300 interaction of myeloid chemokine CCL16 with T cell CXCR6. CXCR6 was highly expressed on $301 R^{2} R_{c}{ }^{+} T$ cells and has been associated with Th17 and $\gamma \delta T 17$ cell recruitment ${ }^{49}$. Together, these 302 data suggest a model in which intrathyroidal $\gamma \delta T 17$ and Th17 activation and induction can occur 303 through: i) binding of PD-1 and CTLA-4 by checkpoint inhibitors, and ii) pro-inflammatory cytokine 304 and chemokine signaling.

$R O R c$ encodes ROR $\gamma \mathrm{t}$, a master transcriptional regulator that drives expression of IL-17 cytokines

307 in multiple cell types ${ }^{50,51}$. Consistent with thyroid infiltration of Rorc ${ }^{+}$immune cells, 308 immunohistochemical staining showed increased IL-17A in thyroid tissue of Dual ICl-treated mice 309 compared to isotype control-treated mice (Fig. 4e). Furthermore, flow cytometric analysis of 310 dissociated thyroid tissue showed increasing numbers of IL-17A-producing $\gamma \delta$ T17 and Th17 cells 311 in ICl-treated mice from 1 to 4 weeks after ICl initiation (Fig. 4f-g). IL-17A-producing CD4- $\gamma \delta$ TCR $^{-}$ 312 T cells were also seen and may represent ILC3 or CD8 ${ }^{+} \mathrm{Tc} 17$ cells $^{52,53}$. Combination ICI therapy 313 produced the greatest infiltration of $\gamma \delta \mathrm{T} 17$ and Th17 cells, followed by single agent anti-PD-1 and 314 anti-CTLA-4 treatment (Fig. $\mathbf{4 g}) \cdot \mathrm{IL}^{-17 \mathrm{~A}^{+}} \gamma \delta$ T cells were also predominantly CD27 ${ }^{-}$and $\mathrm{CD}^{+} 4^{+}$ 315 (Fig. 4h), consistent with scRNAseq data from thyroid infiltrating immune cells and the previously 316 reported phenotype of $\gamma \delta \mathrm{T} 17$ cells $^{54,55}$. Together these data strongly implicate a role for IL-17A 317 ROR $\gamma \mathrm{t}^{+}$Th17 and $\gamma \delta \mathrm{T} 17$ cells in the development of ICl-thyroiditis. 
319 Finally, we sought to identify cellular targets of IL17A, which binds to and signals through 320 IL17RA/IL17RC heterodimers. IL17RA is widely expressed in the body and alone considered 321 insufficient for strong IL-17A signal transduction ${ }^{56}$. IL-17 receptor C (IL-17RC), the necessary 322 binding partner of IL-17RA for IL-17A signaling (Extended Data Fig. 6a), is more selectively 323 expressed ${ }^{56}$. Evaluation of thyroid-infiltrating immune cells $\left(C D 45^{+}\right)$by scRNAseq studies did not 324 show significant expression of IL-17RC (data not shown). However, IL-17RC was expressed on 325 thyroid epithelium in our mouse model by IHC (Extended Data Fig. 6a). In addition, we evaluated

326 IL-17RC gene expression on NThy-Ori-3.1 cells, an SV-40 transformed human thyroid epithelial 327 cell line routinely used to study normal thyroid cell function, by qRT-PCR (Extended Data Fig. 328 6b). NThy-Ori-3.1 cells expressed 4-fold more IL-17RC than 293T cells, a model of human 329 embryonic kidney used as a reference tissue $(p<0.05)$. IL-17A signaling through IL-17RA/RC can 330 induce apoptosis ${ }^{57,58}$. These findings are consistent with a previous report that thyroid follicular 331 epithelial cells express high levels of IL-17RC 59 and suggest that IL17A may directly target thyroid 332 cells.

\section{IL-17A neutralizing antibody reduces autoimmune infiltrates in ICI-treated mice}

335 Based on these data, we predicted that blocking IL-17A could reduce thyroid autoimmunity in ICI336 treated mice. We therefore tested the effects of an IL-17A neutralizing antibody (clone 17F3, $3370.5 \mathrm{mg} /$ dose 3x/week i.p.) 10 days after Dual ICl therapy in NOD mice (Fig. 5a). Indeed, this IL338 17A neutralizing antibody reduced the frequency and severity of autoimmune infiltrates in $\mathrm{ICl}-$ 339 treated mice at 4 weeks, including thyroiditis (Fig. 5b-c; ANOVA p<0.0001, pairwise comparisons 340 vs. Dual ICI $p<0.001$ for isotype, Dual ICI $+\alpha \mathrm{IL}-17 \mathrm{~A}$, and Dual $+\alpha \mathrm{TNF} \alpha)$. Specifically, thyroid 341 infiltrating CD45 ${ }^{+}$immune cells were reduced from mean $16,543 \pm$ SEM 7,940 cells/lobe in Dual 342 ICl-treated mice, to $339 \pm 133$ in Dual ICI with anti-IL17A, and compared to $1,406 \pm 872$ in isotype343 treated mice (ANOVA $p<0.03 ; p$ values vs. Dual ICI were isotype 0.2, Dual + $\alpha$ IL17A 0.16, and 
344 Dual $+\alpha \mathrm{TNF} \alpha \mathrm{CD} 45^{+}$0.26). Thyroid-infiltrating $\mathrm{CD}^{+}(\alpha \beta$ and $\gamma \delta \mathrm{TCR}) \mathrm{T}$ cells were reduced as 345 well, mean $3,418 \pm 1,271$ cells/thyroid lobe in Dual ICI vs. $164 \pm 59$ with Dual ICI + anti-IL17A vs. $346478 \pm 207$ with isotype (ANOVA $p=0.01 ; p$ values for pairwise comparisons vs. Dual ICl were 347 isotype 0.09, Dual $+\alpha$ IL17A 0.05, and Dual $+\alpha$ TNF $\alpha$ CD45 0.36$)$. Dual ICI + anti-IL-17A). Other 348 thyroid-infiltrating immune cell populations evaluated were also decreased, including B cells 349 (mean $212 \pm 77$ cells/thyroid lobe in Dual ICl vs. $20 \pm 10$ in Dual ICI + anti-IL17A vs. $32 \pm 13$ in 350 isotype, ANOVA $p=0.01 ; p$ values $v s$. Dual ICl were isotype 0.08 , Dual $+\alpha$ LL17A 0.06 , Dual + $351 \alpha \mathrm{TNF} \alpha 0.10$ ), NK cells (mean $222 \pm 75$ cells/thyroid lobe in Dual ICl vs. $16 \pm 4$ in Dual ICl + anti352 IL17A vs. $46 \pm 16$ in isotype, ANOVA $p<0.001 ; p$ values $v s$. Dual ICl were isotype 0.09 , Dual + $353 \alpha$ IL17A 0.04 , and Dual $+\alpha \mathrm{TNF} \alpha 0.04$ ), CD11c $\mathrm{c}^{+} \mathrm{CD} 11 \mathrm{~b}^{\text {low }} \mathrm{DC}$ (mean $42 \pm 9$ cells/thyroid lobe in 354 Dual ICl vs. $8 \pm 2$ in Dual ICI + anti-IL17A vs. $11 \pm 3$ in isotype, ANOVA $p<0.001 ; p$ values vs. Dual $355 \mathrm{ICl}$ were isotype 0.008, Dual $+\alpha \mathrm{IL} 17 \mathrm{~A} 0.005$, and Dual $+\alpha \mathrm{TNF} \alpha 0.002)$, and CD11C ${ }^{+} \mathrm{CD} 11 \mathrm{~b}^{+} \mathrm{DC}$ 356 (mean $214 \pm 39$ cells/thyroid lobe in Dual ICI vs. $46 \pm 10$ in Dual ICl + anti-IL17A vs. $67 \pm 13$ in 357 isotype, ANOVA $p<0.001 ; p$ values vs. Dual ICl were isotype 0.006 , Dual $+\alpha$ IL17A 0.002 , and 358 Dual $+\alpha$ TNF $\alpha$ 0.0004). Controls treated with isotype + IL17A inhibitor showed infiltrates 359 comparable to isotype-only controls (data not shown). Interestingly, anti-IL-17A did not 360 significantly reduce thyroid autoantibodies against TPO or Tg (Fig. $\mathbf{5 d}$ ), suggesting that ICl361 associated autoantibody production may be independent from thyroid immune infiltration.

363 Our data also showed thyroid accumulation of macrophages and monocytes that express TNF $\alpha$, 364 a pro-inflammatory cytokine important in the differentiation of $\gamma \delta \mathrm{T} 17$ and Th17 cells ${ }^{42,48}$ (Extended

365 Data Fig. 5a-c). Interestingly TNF $\alpha$ has previously been implicated in patients with ICl-associated 366 colitis; Perez-ruiz et al. ${ }^{22}$ showed that prophylactic TNF $\alpha$ blockade reduced the incidence of colitis 367 during ICl therapy in a mouse model. Given its role in the IL-17A axis and efficacy in preventing $368 \mathrm{ICl}$-associated colitis, we also evaluated the effects of a TNF $\alpha$ neutralizing antibody (clone 
XT3.11, 0.4mg/dose 3x/week i.p.) begun 10 days after anti-PD-1 + anti-CTLA-4 ICI therapy (Fig.

370 5a). A TNF $\alpha$ neutralizing antibody significantly reduced immune infiltrates across multiple tissues

371 in our mouse model of $\mathrm{ICl}$-associated autoimmunity (Fig. $\mathbf{5 b}$ ) and reduced thyroid autoimmune

372 cell infiltrates (Fig. 5c). Thyroid autoantibodies against TPO and Tg were not significantly

373 changed by TNF $\alpha$ neutralizing antibody treatment (Fig. $\mathbf{5 d}$ ). Thus, inhibition of the IL-17A axis

374 (via IL-17A or TNF $\alpha$ blockade) effectively prevents IRAE development with ICI treatment.

375

376

\section{IL-17A neutralizing antibody during ICI treatment does not reduce anti-tumor effects}

377 Our data in ICl-treated NOD mice suggested that a neutralizing IL-17A antibody may reduce IrAE,

378 including ICl-thyroiditis. In developing therapies to prevent or reduce IrAE in patients, it will be

379 critical that any approach preserve or enhance the anti-tumor immune effects of ICl. Recent data

380 from gut IrAE showed that prophylactic TNF $\alpha$ inhibition with a neutralizing antibody during ICI

381 treatment of murine colon cancer model MC38 did not significantly reduce anti-cancer efficacy,

382 as assessed by tumor growth curves and tumor infiltrating effector $T$ cells ${ }^{22}$. Whether IL-17A

383 inhibition will have deleterious effects on the efficacy of $\mathrm{ICI}$ treatment in anti-tumor immunity is 384 unclear, since IL-17A has been associated with pro-tumorigenic effects in some cancers ${ }^{44,60,61}$ 385 and anti-tumorigenic effects in others ${ }^{44,60,61}$. To test this, we determined the effect of IL-17A 386 blockade during ICl treatment on the growth of MC38 tumor cells; this model was selected due to 387 lack of available tumor models in the NOD strain. As expected, dual ICI treatment of MC38 tumors 388 in B6 mice significantly reduced tumor growth (Fig. 5e). Importantly, the addition of an IL-17A 389 neutralizing antibody did not reduce the anti-tumor effect of $\mathrm{ICl}$ treatment and showed a trend 390 toward improved anti-tumor effects (Fig. 5e; ANOVA for day 22 tumor volume $p<0.05 ; p$ values 391 for comparison to isotype were Dual ICI 0.0337, Dual ICI + $\alpha \mathrm{IL}-17 \mathrm{~A}$ 0.008, and $\alpha \mathrm{IL}-17 \mathrm{~A} 0.051$. 392 No significant difference between Dual ICI vs. Dual ICI + $\alpha \mathrm{IL}-17 \mathrm{~A}, \mathrm{p}=0.9)$. In summary, blockade 
of IL-17A function by a neutralizing antibody begun after ICI treatment significantly reduced IrAE 394 while preserving anti-tumor efficacy of anti-PD-1 + anti-CTLA-4 therapy.

\section{Increased circulating IL-17A $T$ cells in ICI-treated patients with thyroid IrAE}

To correlate our findings in mice to humans, we evaluated blood samples of patients with cancer

398 treated with $\mathrm{ICl}$ therapy who developed thyroid IrAE and compared them to subjects with 399 spontaneous thyroid autoimmunity (i.e. Hashimoto's thyroiditis, HT) or no thyroid disease (healthy

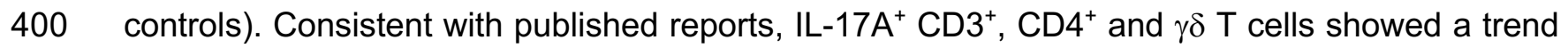
401 for increased frequency in patients with HT compared to controls ${ }^{40,41}$. Furthermore, patients 402 treated with $\mathrm{ICl}$ showed a trend toward increased IL-17A ${ }^{+}$pan-CD3 ${ }^{+}(\alpha \beta$ and $\gamma \delta)$ cells in peripheral 403 blood (Fig. 6a and Extended Data Fig. 7). While CD4 ${ }^{+}$Th17 cells showed a trend toward 404 increase, $\gamma \delta \mathrm{T} 17$ cells were significantly increased in ICl-treated patients compared to healthy 405 controls $(p<0.05)$.

\section{DISCUSSION}

408 Immune checkpoint inhibitors (anti-PD-1/L1 and anti-CTLA-4) have significantly advanced the 409 treatment of cancer since their first approval in 2011. However, the benefits and use of ICI have 410 been limited by the frequent development of unwanted IrAE that contribute to patient morbidity 411 and may lead to interruption of cancer treatment. Despite significant efforts to date, the cause of 412 IrAE remained poorly understood. Using a novel mouse model in which checkpoint inhibitor 413 therapy leads to multi-organ autoimmune infiltrates, we identify Type 3 immune cells including 414 Th17 and $\gamma \delta$ T17 cells as critical contributors to IrAE development in this model (Fig. 6b). PD-1 415 and CTLA-4 are expressed by these immune cell subsets, suggesting that they may be direct 416 targets of $\mathrm{ICl}$. Furthermore, the thyroid microenvironment of ICl-treated mice is replete with TNF $\alpha$, 417 IL1 $\beta$, and other inflammatory cytokines that promote Th17 and $\gamma \delta \mathrm{T} 17$ cell activation and induction. 
418 Indeed, antibody-based inhibition of IL17A and TNF $\alpha$ protected mice from ICI-induced

419 autoimmunity. Finally, IL-17A neutralization with ICI treatment in a tumor model did not reduce

$420 \mathrm{ICl}$ anti-tumor efficacy and indeed showed a trend toward enhancement. Thus, targeting type 3

421 immune cells in ICl-treated patients may reduce IrAE without impairing the anti-tumor efficacy of

$422 \mathrm{ICl}$.

423

424 In our model, ICl-treated NOD mice developed thyroiditis comprised of a T cell predominant but 425 diverse immune infiltrate. Angell et al. first reported on the thyroid immune cell infiltrate in a patient 426 with ICl-thyroiditis, noting lymphocytic cells and histiocytes ${ }^{14}$. In a subsequent study, Kotwal et

427 al. ${ }^{62}$ evaluated immune cells in thyroid FNA specimens from eight ICI-thyroiditis patients and 428 noted a predominant $\mathrm{CD}^{+} \mathrm{T}$ cell population, as well as myeloid cells. Interestingly, they noted a 429 significantly increased $\mathrm{CD}^{-} \mathrm{CD}^{-} \mathrm{CD}^{+} \mathrm{T}$ cell population in $\mathrm{ICl}$-thyroiditis patients compared to 430 healthy controls ( $1.9 \%$ vs. $0.7 \%$ of $C D 45^{+}$cells), though these were not characterized as $\gamma \delta \mathrm{T}$ 431 cells. This study also demonstrated an increase in PD-1 ${ }^{+} \mathrm{T}$ cells in thyroid immune infiltrates 432 compared to healthy controls that is similar to our findings of increased checkpoint protein (PD-1 433 and CTLA-4) expression on thyroid-infiltrating ROR $\gamma \mathrm{t}^{+} \mathrm{T}$ cells in ICl-treated mice. Finally, a recent 434 report by Yasuda et al. $^{26}$ evaluating anti-PD-1 therapy in mice pre-immunized with human 435 thyroglobulin, showed a key role for $\mathrm{CD}^{+} \mathrm{T}$ cells in the development of ICl-associated thyroiditis. 436 While our results are consistent with this, our studies further demonstrate the importance of Th17 437 subsets within $\mathrm{CD} 4^{+} \mathrm{T}$ cells as well as non-CD4 ${ }^{+} \gamma \delta \mathrm{T} 17$ subsets.

439 While Th17 cells are well described in spontaneous autoimmunity, including Hashimoto's 440 thyroiditis, they have not previously been reported in IrAE. $\gamma \delta T 17$ cells, in contrast, have not 441 previously been described in studies of spontaneous thyroiditis or in $\operatorname{IrAE}^{63} \cdot \gamma \delta \mathrm{T} 17$ cells are unique 442 in their early activation and robust cytokine production compared to $\alpha \beta$ TCR T cells ${ }^{42,43,64}$. Given 
443 this, $\gamma \delta \mathrm{T} 17$ cells may underlie the rapid onset that characterizes thyroid IrAEs compared to

444 spontaneous autoimmune thyroiditis. Because $\gamma \delta T 17$ cells have been reported to expand Th17

445 cells, early $\gamma \delta \mathrm{T} 17$ activation with checkpoint inhibition in combination with inflammatory cytokines

446 (e.g. IL-1 $\beta$ and TNF $\alpha$ ) from myeloid cells may drive activation and recruitment of Th17, CD8 ${ }^{+}$, and

447 other T cell populations. Furthermore, other IL-17A producing immune cells, namely ILC3, may

448 also contribute to autoimmunity during $\mathrm{ICI}$ treatment and this possibility warrants further study.

450 The IrAE predisposition of the inbred NOD strain may mimic humans with genetic predisposition 451 to IrAE development. For instance, HLADR15 haplotype has been associated with pituitary $\operatorname{IrAE}^{65}$

452 while the HLADR4 haplotypes has been associated with autoimmune diabetes IrAE in humans ${ }^{20}$.

453 Autoimmune predisposition has been mapped to NOD polymorphic regions which include MHC.

454 This model using NOD mice overcomes some critical limitations of previous models that required 455 deletion of regulatory populations, transfection of human checkpoint proteins, or the requirement 456 for chemical stimulants or xeno-antigen immunization for autoimmunity ${ }^{22-26}$. For instance, 457 NOD.H2h4 mice, which have been used previously to study thyroiditis ${ }^{39,66,67}$ and anti-CTLA-4 458 induced thyroiditis ${ }^{25}$, require excess dietary Nal to precipitate thyroiditis via follicular cell injury 459 and TNF $\alpha$ stimulation, have high background rates of thyroiditis $(100 \%$ after 6 weeks of Nal 460 supplementation ${ }^{66}$ ), and are confounded by the presence of a non-syngeneic MHC class II 461 molecule 27,66 . In contrast, our model in NOD mice provides an immune competent system with 462 low rates of spontaneous autoimmunity at advanced age, more similar to the background human 463 population. As seen in humans, mice with a genetic predisposition (i.e. NOD vs. B6) developed 464 more IrAE across multiple tissues following ICI treatment and with combination vs. single agent $465 \mathrm{ICl}$ therapy ${ }^{20,31}$. 
467 IrAE remain a significant barrier to the use of ICI but therapeutic strategies to prevent IrAEs have 468 not been widely implemented. A primary consideration in the development of strategies to reduce 469 IrAE must be the preservation of anti-cancer effects of $\mathrm{ICI}$. Early efforts using glucocorticoids for 470 IrAE treatment inconsistently reversed autoimmunity or compromised anti-cancer effects ${ }^{5,19}$. In 471 addition, previous studies have shown reduction of thyroid IrAE by elimination of CD4 ${ }^{+} \mathrm{T}_{\text {cells }}{ }^{26}$, 472 but this is not readily translatable to cancer patients given the importance of CD4 ${ }^{+}$T cells in cell473 mediated immunity ${ }^{36,68}$. Similarly, while Tbet $1^{+}$IFN $\gamma$-producing T cells are seen in thyroid immune 474 infiltrates and almost certainly contribute to autoimmunity ${ }^{69}$, these cells are known to be critical to 475 anti-cancer effects of $\mathrm{ICl}^{46,70,71}$ and therefore cannot feasibly be inhibited for IrAE prevention. 476 Here, we provide additional evidence that TNF blockade may prevent $\operatorname{IrAE}^{22}$ and also suggest 477 that IL-17 blockade may be useful. Both targets have therapies that are already FDA approved. 478 While focused on thyroid IrAE, a common autoimmune side effect encountered during $\mathrm{ICI}$ 479 treatment that results in permanent organ dysfunction, these mechanisms may be shared among 480 IrAE in other organs with more grave clinical consequences. ICl-associated colitis has been 481 associated with increased circulating IL-17A in patients treated with anti-CTLA-4 ${ }^{72,73}$. Mice in our 482 model developed multi-organ autoimmunity, including colitis, pneumonitis, hepatitis, and 483 nephritis, and therefore can be used to study IrAE in these organs. Furthermore, neutralizing IL484 17A antibody therapy decreased overall autoimmune infiltrates, including in non-thyroid tissues, 485 and therefore may be of utility in reducing IrAE during ICI more broadly. Based upon these results, 486 and the prior important work of others ${ }^{22}$, we propose that a clinical trial evaluating inhibition of the 487 IL-17A axis for prevention of IrAE in patients with cancer receiving ICI treatment should be 488 pursued. 
$491 \quad$ None

Figure Legends

Figure 1. ICl therapy induces multi-system autoimmune infiltrates (IrAE) in B6 and NOD mice.

a, Schematic of ICl drug treatment. b, Comparison of autoimmune organ infiltration after 4 weeks of $\mathrm{ICl}$ vs. isotype treatment in C57/B6 (B6) mice ( $n=8$ isotype, $n=8$ Dual ICl); Pie charts (right) show tissue infiltrate for each mouse; black $=$ immune infiltrate, white $=$ no infiltrate, gray $=$ no data. c, Quantification of anti-thyroid autoantibodies in B6 mice after 4 weeks of isotype $(n=8)$ or Dual $\mathrm{ICI}(n=8)$ treatment. $d$, Comparison of autoimmune organ infiltration after 8 weeks of $\mathrm{ICI}$ treatment in NOD mice ( $n=12$ isotype, $\mathrm{n}=11$ anti-CTLA-4, $n=11$ anti-PD-1, and $n=13$ Dual ICI). Pie charts show tissue infiltrate for each mouse, as in b above. e, Representative H\&E micrographs of autoimmune tissue infiltrates in Dual ICl vs. isotype treated mice (original mag.

504 100x). f, Quantification of anti-thyroid autoantibodies in NOD mice after 8 weeks of treatment 505 ( $n=10$ isotype anti-Tg or 13 for anti-TPO, $n=12$ anti-CTLA-4, $n=11$ anti-PD-1, and $n=13$ Dual ICI 506 for anti-Tg or 22 for anti-TPO). g, Free thyroxine serum levels in ICl-treated NOD mice after 8 507 weeks of treatment. Data are mean \pm SEM. ${ }^{*} p<0.05,{ }^{* *} p<0.01,{ }^{* *} p<0.001$, unpaired t test, two508 tailed (b,c), Brown-Forsythe ANOVA, assuming unequal s.d., followed by Dunnett's multiple 509 comparisons test $(\mathbf{d}, \mathbf{g})$, or one-way ANOVA and Dunnett's multiple comparison test (f).

511 Figure 2. Flow cytometry analysis of peripheral immune changes in ICl-treated NOD mice.

512 a, Frequency of $\mathrm{CD}^{+} \mathrm{T}\left(\mathrm{CD}^{+} \mathrm{NKp}^{-}\right)$cells in spleen in isotype $(n=7)$ vs. anti-PD-1 + anti-CTLA5134 (Dual ICI, n=8) NOD mice after 4 weeks. b, Relative frequency of activated T cells (CD44 $\left.514 \mathrm{CD}_{2} \mathrm{~L}^{-}\right)$among $\mathrm{CD}^{+}$and $\mathrm{CD}^{+} \mathrm{T}$ cell subsets in spleen in isotype $(n=7$ or 8$)$ and Dual ICI $(n=8$ 515 or 10). c, Relative frequency of interferon (IFN)- $\gamma^{+}$cells within CD4 ${ }^{+}$population between isotype 
$516(n=7)$ and Dual ICl-treated $(n=8)$ mice. d, Relative frequency of $\mathrm{IL}_{-17 A^{+}}$cells in CD4 ${ }^{+}$pop.

517 between isotype $(n=14)$ and Dual ICl-treated $(n=14)$ mice. e, Representative histogram of ROR $\gamma \mathrm{t}^{+}$

518 by thyroid-infiltrating $\mathrm{CD}^{+}$cells from isotype vs. ICl-treated mice; $7 \mathrm{ICl}$ treated and 8 isotype mice

519 evaluated. f, Gating strategy for $\mathrm{CD}^{+}$and $\mathrm{IL}^{-17 \mathrm{~A}^{+}}$populations from spleen, and relative

520 frequency of $\mathrm{CD}^{+}$and $\mathrm{CD} 4^{-} \mathrm{IL}-17 \mathrm{~A}^{+}$cells in spleen from isotype $(n=12)$ and Dual ICI-treated

$521(n=10)$ mice. $\mathbf{g}$, Frequency of IL-17A cells within gamma delta $(\gamma \delta)$ cells in spleen from isotype

$522(n=12)$ and Dual ICl-treated $(n=10)$ mice. Data are mean. ${ }^{*} p<0.05$, two-tailed, unpaired t test with

523 Welch correction, assuming unequal s.d., and Holm-Sidak method correction for multiple

524 comparisons (a-d,f,g).

525

526 Figure 3. Characterization of thyroid-infiltrating immune cells in ICI-treated NOD mice.

527 a, IHC staining for T cells (CD3), macrophages (F4/80), and B cells (B220) in thyroid tissue from

528 Dual ICl- $(n=7,10$, or 9$)$ vs. isotype- $(n=4)$ treated mice [left, representative sections, $400 x$ original

529 mag.; right, cells/hpf]. b, Representative gating strategies and dot plots (left) and relative

530 frequency of $\mathrm{CD} 45^{+}$immune cells in thyroid tissue (right, cells per thyroid lobe) from isotype $(n=11)$

531 vs. Dual ICI $(n=17)$ treated mice after 4 weeks. c, Relative frequency of immune subpopulations

532 in isotype $\left(n=11\right.$, except $n=16$ for $\mathrm{CD}^{+}$and $\left.\mathrm{B}^{2} 20^{+}\right)$vs. Dual ICl-treated $(n=17$, except $n=23$ for

$533 \mathrm{CD}^{+}$and $\mathrm{B}^{2} 20^{+}$) mice. $\mathbf{d}$, Schematic of tissue processing for single cell RNA sequencing (left).

534 UMAP plot of 4,095 thyroid-infiltrating CD45 ${ }^{+}$immune cells from 10 pooled Dual ICl-treated mice,

5352 independent experiments (right). Cluster analysis yields 14 distinct clusters comprising CD4,

536 CD8, and $\gamma \delta \mathrm{T}$ cells, natural killer (NK)/NKT cells, innate lymphoid cells (ILC), B cells,

537 macrophages, and dendritic (DC)/antigen presenting cells (APC). e, Intrathyroidal gene

538 expression for $C d 45$ and $C d 3 e$ in ICl-treated vs. isotype mice measured by qRT-PCR. f, UMAP

539 of CD3-subclustered cells, $n=3,178$ cells. Cluster analysis yields 10 distinct clusters comprising

540 CD4, CD8, and $\gamma \delta$ T cells (left). Feature and violin plots for T cell subset markers for $\delta \gamma(T r d c)$, 
541 CD4 (Cd4), and CD8 (Cd8a) (right). g, Violin plots for type 3 immunity (RORyt-pathway)

542 associated genes Rorc and II23r. h, Intrathyroidal gene expression for Rorc, II23r, and II17a in

543 ICl-treated vs. isotype mice, by qRT-PCR. i, Comparison of thyroid-infiltrating ROR $\gamma \mathrm{t}^{+}$pan-CD3 ${ }^{+}$

$544(\alpha \beta$ and $\gamma \delta)$ T cells by flow cytometry in ICl-treated $(n=8)$ vs. isotype $(n=6)$ mice. Data are

545 mean \pm SEM. For qRT-PCR, mean fold change \pm SEM, $n=12$ animals/group; thyroid tissue pooled

546 and run in 3 experiments, in triplicate. ${ }^{*} p<0.05,{ }^{*} p<0.01$, two-tailed, unpaired $t$ test with Welch

547 correction, assuming unequal s.d. (a-c,i) and Holm-Sidak method correction for multiple

548 comparisons $(\mathbf{e}, \mathbf{h})$.

549

550 Figure 4. RORyt ${ }^{+} \mathrm{IL}-17 \mathrm{~A}^{+} \mathrm{T}$ cells in thyroid immune infiltrates of ICl-treated mice.

551 a, UMAP of Rorc-subclustered cells, $n=361$ cells. Cluster analysis yields 3 distinct clusters (left)

552 comprised of CD4 and $\gamma \delta$ T cells, as shown by violin plots (right). b. Feature and violin plots for

553 Th17- and $\gamma \delta$ T17-associated genes. c-d, Violin plots for checkpoint proteins (c) Pdcd1 and Ctla4

554 and inflammatory cytokine receptors (d) Tnfrsf1a and I/1r1 on Rorc thyroid-infiltrating T cells. e,

555 IHC staining for IL-17A in thyroid tissue of isotype and Dual ICl-treated mice (representative 556 sections from 3 independent experiments, original mag. 400x). f, Gating strategy and 557 representative dot plots of IL-17A $\mathrm{A}^{+} \mathrm{CD} 4+(\mathrm{Th} 17)$ and $\gamma \delta^{+}(\gamma \delta \mathrm{T} 17)$ cells in thyroid immune infiltrates 558 in isotype or Dual ICl-treated mice at $1-4$ weeks ( $n=2$ pooled animals each condition). $\mathbf{g}$, 559 Quantification of intrathyroidal $\mathrm{CD} 4^{+} \mathrm{IL}^{-17 \mathrm{~A}^{+}}$and $\mathrm{TCR} \gamma \delta^{+} \mathrm{IL}-17 \mathrm{~A}^{+}$cells from thyroid immune 560 infiltrates from ICl-treated mice at different timepoints of ICl-thyroiditis development. $\mathbf{h}$, 561 Representative gating strategy and flow cytometry dot plots showing $\mathrm{CD} 44^{+}$and $\mathrm{CD} 27^{-}$phenotype 562 of TCR $\gamma \delta^{+} I L-17 A^{+}$cells. $g-h$, for plots shown, pooled thyroid immune infiltrates from isotype $(n=2)$,

563 anti-PD-1 $(n=3)$, anti-CTLA-4 $(n=4)$, and Dual ICI $(n=2)$ treated mice after 4 weeks, or sooner 564 timepoints $(n=2)$ from Dual ICI treated for Time Course. 


\section{ICl)-associated autoimmune infiltrates.}

568 a, Schematic of treatment regimen. b, Autoimmune organ infiltrate score after 4 weeks of $\mathrm{ICl}$ 569 treatment in isotype $(n=14)$, Dual ICI $(n=16)$, Dual ICI + neutralizing IL-17A antibody ( $\alpha$ IL17A, $570 n=7)$, and Dual ICI + neutralizing TNF $\alpha$ antibody ( $\alpha \mathrm{TNF} \alpha, n=8)$. Pie charts $($ right $)$ showing tissues 571 with immune infiltration after 4 weeks of $\mathrm{ICI}$ treatment. Each pie represents one animal; black = 572 immune infiltrate, white $=$ no infiltrate, gray $=$ no data. c, Intrathyroidal immune cell frequency 573 among groups [isotype, $n=11$, except $n=16$ for $\left.\mathrm{CD}^{+}\right)$, Dual $\mathrm{ICl}\left(n=17\right.$, except $n=23$ for $\left.\mathrm{CD}^{+}\right)$,

574 Dual ICI $+\alpha \operatorname{IL17A}(n=8)$, Dual ICI $+\alpha \operatorname{TNF} \alpha(n=8)]$. d, Anti-thyroid autoantibodies in NOD mice 575 after 4 weeks of treatment with isotype ( $n=14$ for anti-TPO, $n=12$ for anti-Tg), Dual ICl $(n=18$ for 576 anti-TPO, n=13 for anti-Tg), Dual ICI + $\alpha \mathrm{IL} 17 \mathrm{~A}(n=8)$, Dual ICI $+\alpha \mathrm{TNFa}(n=8)$. e, Growth of MC38 577 tumors in B6 mice treated with anti-PD-1 + anti-CTLA-4 (Dual ICI) or isotype control, as well as a 578 neutralizing IL-17A ( $\alpha$ IL17A) or relevant isotype, time ( $n=6$ each group). Data are mean \pm SEM 579 shown. ${ }^{* *} p<0.01,{ }^{* * *} p<0.001,{ }^{* * * *} p<0.0001$. Brown-Forsythe ANOVA, assuming unequal s.d., 580 followed by Dunnett's multiple comparisons test as indicated (b-d), or ANOVA for tumor volume 581 at day 22, followed by Dunnett's multiple comparisons test (e).

\section{$583 \quad$ Figure 6. $\gamma \delta \mathrm{T} 17$ and Th17 cells in ICl-treated cancer patients.}

584 a, Relative frequency of IL-17A ${ }^{+}$pan-CD3 ${ }^{+}$, Th17, and $\gamma \delta \mathrm{T} 17$ cells in peripheral blood of controls $585(n=6)$, patients with Hashimoto's thyroiditis (HT, $n=13)$, or patients with cancer treated with $\mathrm{ICI}$ 586 ( $n=20)$. Mean shown. ${ }^{*} \mathrm{p}<0.05$. Brown-Forsythe ANOVA, assuming unequal s.d., followed by 587 Dunnett's multiple comparisons test. b, Proposed mechanism of Thyroid IrAE. (1) ROR $\gamma \mathrm{t}^{+}$Th17 588 and $\gamma \delta$ T17 cells may be activated by anti-PD-1 and/or anti-CTLA-4 inhibitor binding to (2) release 589 IL-17A. (3) In addition, myeloid cells within the thyroid may contribute to Th17 and $\gamma \delta T 17$ activation 590 and recruitment by production inflammatory cytokines IL-1 $\beta$ and TNF $\alpha$ and chemokines. IL-17A 
within the thyroid microenvironment may affect thyroid follicular cell death via (4) induction of apoptosis or indirectly by (5) facilitating the accumulation of cytotoxic effector cells. (6) Strategies

593 to reduce IrAE target the IL-17A axis.

594

595

596

597

598

599

600

601

602

603

604

605

606

607

608

609

610

611

612

613

614

615

616

617

618

619

620

621

622

623

624

625

626

627

628

629

630

631

632

633

634

635

636

637

638

\section{References Cited}

1. Wei, S. C., Duffy, C. R. \& Allison, J. P. Fundamental mechanisms of immune checkpoint blockade therapy. Cancer Discov. 8, 1069-1086 (2018).

2. Haslam, A. \& Prasad, V. Estimation of the Percentage of US Patients With Cancer Who Are Eligible for and Respond to Checkpoint Inhibitor Immunotherapy Drugs. JAMA Netw. open 2, e192535 (2019).

3. Larkin, J. et al. Five-year survival with combined nivolumab and ipilimumab in advanced melanoma. N. Engl. J. Med. 381, 1535-1546 (2019).

4. Arnaud-Coffin, P. et al. A systematic review of adverse events in randomized trials assessing immune checkpoint inhibitors. International Journal of Cancer vol. 145 639_ 648 (2019).

5. Das, S. \& Johnson, D. B. Immune-related adverse events and anti-tumor efficacy of immune checkpoint inhibitors. J. Immunother. Cancer 7, 1-11 (2019).

6. De Filette, J., Andreescu, C. E., Cools, F., Bravenboer, B. \& Velkeniers, B. A Systematic Review and Meta-Analysis of Endocrine-Related Adverse Events Associated with Immune Checkpoint Inhibitors. Hormone and Metabolic Research vol. 51 145-156 (2019).

7. Wang, D. Y. et al. Fatal Toxic Effects Associated With Immune Checkpoint Inhibitors: A Systematic Review and Meta-analysis. JAMA Oncol. 4, 1721-1728 (2018).

8. Young, A., Quandt, Z. \& Bluestone, J. A. The balancing act between cancer immunity and autoimmunity in response to immunotherapy. Cancer Immunol. Res. 6, 1445-1452 (2018).

9. Iyer, P. C. et al. Immune-Related Thyroiditis with Immune Checkpoint Inhibitors. Thyroid 28, 1243-1251 (2018).

10. Delivanis, D. A. et al. Pembrolizumab-induced thyroiditis: Comprehensive clinical review and insights into underlying involved mechanisms. J. Clin. Endocrinol. Metab. 102, 27702780 (2017).

11. De Moel, E. C. et al. Autoantibody development under treatment with immune-checkpoint inhibitors. Cancer Immunol. Res. 7, 6-11 (2019).

12. Ma, C. et al. The impact of high-dose glucocorticoids on the outcome of immunecheckpoint inhibitor-related thyroid disorders. Cancer Immunol. Res. 7, 1214-1220 (2019).

13. Kotwal, A. et al. Immune Checkpoint Inhibitor-Induced Thyroiditis Is Associated with Increased Intrathyroidal T Lymphocyte Subpopulations. Thyroid 30, 1440-1450 (2020).

14. Angell, T. E., Min, L., Wieczorek, T. J. \& Hodi, F. S. Unique cytologic features of thyroiditis caused by immune checkpoint inhibitor therapy for malignant melanoma. Genes Dis. 5, 46-48 (2018).

15. Nakamura, Y. Biomarkers for Immune Checkpoint Inhibitor-Mediated Tumor Response and Adverse Events. Front. Med. 6, (2019).

16. Álvarez-Sierra, D. et al. Analysis of the PD-1/PD-L1 axis in human autoimmune thyroid disease: Insights into pathogenesis and clues to immunotherapy associated thyroid autoimmunity. J. Autoimmun. 103, (2019).

17. Brahmer, J. R. et al. JOURNAL OF CLINICAL ONCOLOGY. J Clin Oncol 36, 1714-1768 (2018). 
639

640

641

642

643

644

645

646

647

648

649

650

651

652

653

654

655

656

657

658

659

660

661

662

663

664

665

666

667

668

669

670

671

672

673

674

675

676

677

678

679

680

681

682

683

684

685

686

687

688

689

18. Thompson, J. A. et al. NCCN Guidelines Insights: Management of ImmunotherapyRelated Toxicities, Version 1.2020. J. Natl. Compr. Canc. Netw. 18, 230-241 (2020).

19. Adam, K., luga, A., Tocheva, A. S. \& Mor, A. A novel mouse model for checkpoint inhibitor-induced adverse events. PLoS One 16, 1-14 (2021).

20. Bluestone, J. A. et al. Collateral damage: Insulin-dependent diabetes induced with checkpoint inhibitors. in Diabetes vol. 67 1471-1480 (American Diabetes Association Inc., 2018).

21. Faje, A. T. et al. High-dose glucocorticoids for the treatment of ipilimumab-induced hypophysitis is associated with reduced survival in patients with melanoma. Cancer 124, 3706-3714 (2018).

22. Perez-Ruiz, E. et al. Prophylactic TNF blockade uncouples efficacy and toxicity in dual CTLA-4 and PD-1 immunotherapy. Nature vol. 569 428-432 (2019).

23. Liu, J. et al. Assessing immune-related adverse events of efficacious combination immunotherapies in preclinical models of cancer. Cancer Res. 76, 5288-5301 (2016).

24. Du, X. et al. Uncoupling therapeutic from immunotherapy-related adverse effects for safer and effective anti-CTLA-4 antibodies in CTLA4 humanized mice. Cell Res. 28, 433-447 (2018).

25. Sharma, R., Di Dalmazi, G. \& Caturegli, P. Exacerbation of Autoimmune Thyroiditis by CTLA-4 Blockade: A Role for IFNy-Induced Indoleamine 2, 3-Dioxygenase. Thyroid 26, 1117-1124 (2016).

26. Yasuda, Y. et al. CD4 + T cells are essential for the development of destructive thyroiditis induced by anti - PD-1 antibody in thyroglobulin-immunized mice. 7495, (2021).

27. McLachlan, S. M. et al. Genes outside the major histocompatibility complex locus are linked to the development of thyroid autoantibodies and thyroiditis in NOD.H2h4 mice. Endocrinology 158, 702-713 (2017).

28. Quintana, F. J. \& Cohen, I. R. Autoantibody patterns in diabetes-prone NOD mice and in standard C57BL/6 mice. J. Autoimmun. 17, 191-197 (2001).

29. Jiang, W., Anderson, M. S., Bronson, R., Mathis, D. \& Benoist, C. Modifier loci condition autoimmunity provoked by Aire deficiency. J. Exp. Med. 202, 805-815 (2005).

30. Toi, Y. et al. Profiling Preexisting Antibodies in Patients Treated with Anti-PD-1 Therapy for Advanced Non-Small Cell Lung Cancer. JAMA Oncol. 5, 376-383 (2019).

31. Yamazaki, H. et al. Potential risk factors for nivolumab-induced thyroid dysfunction. In Vivo (Brooklyn). 31, 1225-1228 (2017).

32. Anderson, M. S. \& Bluestone, J. A. THE NOD MOUSE: A Model of Immune Dysregulation. Annu. Rev. Immunol. 23, 447-485 (2005).

33. Podolin, P. L. et al. I-E+ nonobese diabetic mice develop insulitis and diabetes. J. Exp. Med. 178, 793-804 (1993).

34. Damotte, D. et al. Analysis of susceptibility of NOD mice to spontaneous and experimentally induced thyroiditis. Eur. J. Immunol. 27, 2854-2862 (1997).

35. Su, M. A. et al. Mechanisms of an autoimmunity syndrome in mice caused by a dominant mutation in Aire. J. Clin. Invest. 118, 1712-1726 (2008).

36. Oh, D. Y. et al. Intratumoral CD4+ T Cells Mediate Anti-tumor Cytotoxicity in Human Bladder Cancer. Cell 181, 1612-1625.e13 (2020).

37. Yost, K. E. et al. Clonal replacement of tumor-specific T cells following PD-1 blockade. 25, 1251-1259 (2020).

38. Miossec, P., Korn, T. \& Kuchroo, V. K. Interleukin-17 and type 17 helper T cells. N. Engl. J. Med. 361, 888 (2009).

39. Horie, I. et al. T helper type 17 immune response plays an indispensable role for development of iodine-induced autoimmune thyroiditis in nonobese diabetic-H2h4 mice. Endocrinology 150, 5135-5142 (2009).

40. Shi, Y. et al. Differentiation imbalance of Th1/Th17 in peripheral blood mononuclear cells 
might contribute to pathogenesis of Hashimoto's thyroiditis. Scand. J. Immunol. 72, 250255 (2010).

41. Konca Degertekin, C. et al. Circulating Th17 cytokine levels are altered in Hashimoto's thyroiditis. Cytokine 80, 13-17 (2016).

42. Papotto, P. H., Reinhardt, A., Prinz, I. \& Silva-Santos, B. Innately versatile: $ү \delta 17$ T cells in inflammatory and autoimmune diseases. J. Autoimmun. 87, 26-37 (2018).

43. Papotto, P. H., Ribot, J. C. \& Silva-Santos, B. IL-17 + yס T cells as kick-starters of inflammation. Nat. Immunol. 18, 604-611 (2017).

44. Kuen, D. S., Kim, B. S. \& Chung, Y. Il-17-producing cells in tumor immunity: Friends or foes? Immune Netw. 20, 1-20 (2020).

45. Stuart, T. et al. Comprehensive Integration of Single-Cell Data. Cell 177, 1888-1902.e21 (2019).

46. Ayers, M. et al. IFN- - -related mRNA profile predicts clinical response to PD-1 blockade. J. Clin. Invest. 127, 2930-2940 (2017).

47. Tan, L. et al. Single-Cell Transcriptomics Identifies the Adaptation of Scart1+ Vy6+ T Cells to Skin Residency as Activated Effector Cells. Cell Rep. 27, 3657-3671.e4 (2019).

48. Veldhoen, M., Hocking, R. J., Atkins, C. J., Locksley, R. M. \& Stockinger, B. TGF $\beta$ in the context of an inflammatory cytokine milieu supports de novo differentiation of IL-17producing T cells. Immunity 24, 179-189 (2006).

49. Butcher, M. J., Wu, C. I., Waseem, T. \& Galkina, E. V. CXCR6 regulates the recruitment of pro-inflammatory IL-17A-producing T cells into atherosclerotic aortas. Int. Immunol. 28, 255-261 (2016).

50. Barros-Martins, J. et al. Effector $y \delta$ T Cell Differentiation Relies on Master but Not Auxiliary Th Cell Transcription Factors. J. Immunol. 196, 3642-3652 (2016).

51. Ivanov, I. I. et al. The orphan nuclear receptor RORgammat directs the differentiation program of proinflammatory IL-17+ T helper cells. Cell 126, 1121-1133 (2006).

52. Liang, Y., Pan, H. F. \& Ye, D. Q. Tc17 cells in immunity and systemic autoimmunity. Int. Rev. Immunol. 34, 318-331 (2015).

53. Kuo, T.-C. et al. Ultrasonographic features for differentiating follicular thyroid carcinoma and follicular adenoma. Asian J. Surg. 43, 339-346 (2020).

54. Cai, Y. et al. Pivotal Role of Dermal IL-17-Producing yס T Cells in Skin Inflammation. Immunity 35, 596-610 (2011).

55. Markle, J. G. M. et al. yठ T Cells Are Essential Effectors of Type 1 Diabetes in the Nonobese Diabetic Mouse Model. J. Immunol. 190, 5392-5401 (2013).

56. Nies, J. F. \& Panzer, U. IL-17C/IL-17RE: Emergence of a Unique Axis in TH17 Biology. Front. Immunol. 11, 341 (2020).

57. Bockerstett, K. A. et al. Interleukin-17A Promotes Parietal Cell Atrophy by Inducing Apoptosis. Cmgh 5, 678-690.e1 (2018).

58. Su, S. an et al. Interleukin-17A mediates cardiomyocyte apoptosis through Stat3-iNOS pathway. Biochim. Biophys. Acta - Mol. Cell Res. 1863, 2784-2794 (2016).

59. Uhlén, M. et al. Proteomics. Tissue-based map of the human proteome. Science 347, 1260419 (2015).

60. Fabre, J. et al. Targeting the tumor microenvironment: The protumor effects of IL-17 related to cancer type. Int. J. Mol. Sci. 17, (2016).

61. Zhao, J., Chen, X., Herjan, T. \& Li, X. The role of interleukin-17 in tumor development and progression. J. Exp. Med. 217, 1-13 (2020).

62. Kotwal, A. et al. Immune Checkpoint Inhibitor-Induced Thyroiditis Is Associated with Increased Intrathyroidal T Lymphocyte Subpopulations. Thyroid 00, 1-11 (2020).

63. Paolieri, F. et al. Infiltrating y/gd T-cell receptor-positive lymphocytes in Hashimoto's thyroiditis, Graves' disease and papillary thyroid cancer. J. Endocrinol. Invest. 18, 295298 (1995). 
741

742

743

744

745

746

747

748

749

750

751

752

753

754

755

756

757

758

759

760

761

762

763

764

765

766

767
64. Sutton, C. E. et al. Interleukin-1 and IL-23 Induce Innate IL-17 Production from y $\delta$ T Cells, Amplifying Th17 Responses and Autoimmunity. Immunity 31, 331-341 (2009).

65. Yano, S. et al. Human leucocyte antigen DR15, a possible predictive marker for immune checkpoint inhibitor-induced secondary adrenal insufficiency. Eur. J. Cancer 130, 198$203(2020)$.

66. Braley-Mullen, H., Sharp, G. C., Medling, B. \& Tang, H. Spontaneous Autoimmune Thyroiditis in NOD.H-2h4 Mice. Journal of Autoimmunity vol. 12 http://www.idealibrary.comon (1999).

67. McLachlan, S. M., Aliesky, H. A. \& Rapoport, B. Aberrant lodine Autoregulation Induces Hypothyroidism in a Mouse Strain in the Absence of Thyroid Autoimmunity. J. Endocr. Soc. 2, 63-76 (2018).

68. Tay, R. E., Richardson, E. K. \& Toh, H. C. Revisiting the role of CD4+ T cells in cancer immunotherapy-new insights into old paradigms. Cancer Gene Ther. 28, 5-17 (2021).

69. Horie, I., Abiru, N., Sakamoto, H., Iwakura, Y. \& Nagayama, Y. Induction of autoimmune thyroiditis by depletion of CD4 +CD25 + regulatory T cells in thyroiditis-resistant IL-17, but not interferon-y receptor, knockout nonobese diabetic-H2 h4 mice. Endocrinology 152, 4448-4454 (2011).

70. Gao, J. et al. Loss of IFN-y Pathway Genes in Tumor Cells as a Mechanism of Resistance to Anti-CTLA-4 Therapy. Cell 167, 397-404.e9 (2016).

71. Shi, L. Z. et al. Interdependent IL-7 and IFN-y signalling in T-cell controls tumour eradication by combined $\alpha-C T L A-4+\alpha-P D-1$ therapy. Nat. Commun. 7, (2016).

72. Tarhini, A. A. et al. Baseline circulating IL-17 predicts toxicity while TGF- $\beta 1$ and IL-10 are prognostic of relapse in ipilimumab neoadjuvant therapy of melanoma. J. Immunother. Cancer 3, 15-20 (2015).

73. von Euw, E. et al. CTLA4 blockade increases Th17 cells in patients with metastatic melanoma. J. Transl. Med. 7, 1-13 (2009). 


\section{METHODS}

769 Mice

770 NOD/ShiLtJ (NOD) and C57/B6 (B6) mice were obtained from the Jackson Laboratory. Male and

771 female mice were used in equal proportions unless otherwise specified. Mice were used at 4-6-

772 week of age unless otherwise noted. Mice were housed in a specific pathogen-free barrier facility

773 at the University of California Los Angeles. Diabetes, determined by presence of glucose in urine

774 (Diastix, Bayer), was assessed at least once per week and diabetic mice were treated daily with

775 intraperitoneal insulin as described previously ${ }^{35}$ until used in experiments or euthanized. All

776 experiments were conducted under IACUC-approved protocols and complied with the Animal

777 Welfare Act and the National Institutes of Health guidelines for the ethical care and use of animals

778 in biomedical research.

$780 \quad$ Cell lines

781 Cell lines used in these studies included MC38, a murine colon tumor model on the B6

782 background; NThyOri-3.1, a human thyroid follicular cell line generated by SV40 transformation

783 of normal thyroid cells; 293 T cells, a human embryonic kidney cell line; and CHO-mTPO, Chinese

784 hamster ovary cells stably transfected for surface membrane expression of mouse thyroid 785 peroxidase (TPO) protein expression ${ }^{67}$. Tumor cell lines were obtained from the American Type 786 Culture Collection [(ATCC), MC38 and NThyOri-3.1)] or were gifted to Dr. Lechner or Dr. Su 787 (CHO-mTPO cells by Drs. Basil Rapoport and Sandra McLachlan; 293T cells by Dr. Christopher 788 Seet; MC38 cells by Dr. Antoni Ribas). Tumor cell line authenticity was performed by surface 789 marker analysis performed at ATCC or in our laboratory. MC38, NThyOri-3.1 and 293T cells were 790 grown in complete medium [RPMI-1640 supplemented with $10 \%$ fetal bovine serum (FBS), 2 mM

791 L-glutamine, 1mM HEPES, non-essential amino acids, and antibiotics (100 U/mL Penicillin and

$792100 \mu \mathrm{g} / \mathrm{mL}$ Streptomycin)], at $37^{\circ} \mathrm{C}$ in humidified, $5 \% \mathrm{CO}_{2}$ incubators. CHO-mTPO cells were 793 cultured in F12 media supplemented with 10\% FBS, 2mM L-glutamine, 1mM HEPES, non- 
794 essential amino acids, and antibiotics (100 U/mL Penicillin and $100 \mu \mathrm{g} / \mathrm{mL}$ Streptomycin). Cell

795 lines were monitored regularly for phenotype. Early passage cells were used for experiments (P1 796 or $\mathrm{P} 2)$.

798 Reagents and media

799 Immune checkpoint inhibitor antibodies used were anti-mouse PD1 (clone RPM1-14), CTLA-4 800 (clone 9D9), and isotype controls [clone 2A3 and MPC-11, respectively] (BioXcell). For inhibitor 801 experiments, a neutralizing antibody against mouse interleukin-17A (clone 17F3) and mouse 802 tumor necrosis factor a (clone XT3.11), from BioXCell. Antibodies were diluted in sterile PBS for 803 use. Human recombinant cytokines IL-17A and TNF $\alpha$ from PeproTech and R\&D Systems, 804 respectively, were reconstituted and stored per manufacturer instructions. For in vitro 805 experiments, primary immune cells were cultured in RPMI complete media [supplemented with $80610 \%$ fetal bovine serum (FBS), $2 \mathrm{mM}$ L-glutamine, $1 \mathrm{mM}$ HEPES, non-essential amino acids, and 807 antibiotics (penicillin and streptomycin)], with 50uM beta-mercaptoethanol.

Immune checkpoint inhibitor treatment of mice

810 Groups of 4-6 week old NOD or B6 mice were used for ICI inhibitor experiments. Mice were 811 randomized to twice weekly treatment with anti-mouse CTLA-4 (clone 9D9), or PD-1 (RPM1-14), 812 both anti-CTLA-4 and anti-PD-1, or an isotype control (2A3, MPC-11), at $10 \mathrm{mg} / \mathrm{kg} / \mathrm{dose}$ 813 intraperitoneally (i.p.) for four or eight weeks. During treatment mice were monitored daily for 814 activity (including signs of neuropathy) and appearance, and twice weekly for weight and 815 glucosuria. Mice developing glucosuria were treated with 10 units of subcutaneous NPH insulin 816 daily. Thyroid dysfunction was assessed by measurement of free thyroxine (FT4) in sera by ELISA 817 (LSBio). After four or eight weeks of ICI treatment (as indicated), mice were euthanized, blood 818 collected by retro-orbital bleed, then perfused with $10 \mathrm{~mL}$ of phosphate buffered saline (PBS). 819 Tissues were collected for histologic analysis into neutral buffered formalin, including thyroid, 
820 lacrimal and salivary glands, lung, liver, kidney, heart, colon, eye, gonad, and pancreas. Serum

821 was collected from blood (centrifugation 1000xg for $30 \mathrm{~min}$ ). Predetermined endpoints for

822 euthanasia before four weeks included $>20 \%$ weight loss and glucosuria not resolved by insulin

823 therapy, as per IACUC protocols.

824

825 Histology

826 Harvested organs were fixed in $10 \%$ buffered formalin for at least 96 hours and then stored in

$82770 \%$ ethanol. Organs were embedded in paraffin, sectioned $(4 \mu \mathrm{m})$, and stained with hematoxylin

828 and eosin (H\&E) by the UCLA Translational Pathology Core Laboratory. Immune infiltration was

829 assessed on H\&E sections using an adapted immune infiltrate scoring system reported

830 previously ${ }^{35}$. The presence of interstitial inflammation, lymphocytic aggregates, perivascular

831 inflammation, follicular disruption, and Hurthle cell change was assessed as either present or

832 absent on H\&E tissue sections by a clinical pathologist (E.D.R.). In addition, each tissue was

833 given an aggregate immune infiltrate score of 0 (no immune infiltrate), 1 (1-2 focal areas of

834 immune infiltrate and/or sparse interstitial inflammation), 2 [ $>2$ focal areas of immune infiltration,

835 and/or presence of both focal glomerulonephritis and perivascular immune infiltration (kidney),

836 and/or diffuse immune infiltrate affecting $>25-49 \%$ of the tissue area] or 3 (diffuse immune infiltrate

837 affecting $>50 \%$ of the tissue area). For pancreas tissue, immune infiltrate score was modified as

8380 for no immune infiltrate, 1 for $<50 \%$ of islets affected by immune infiltration, or 2 for $>50-100 \%$

839 of islets affected by immune infiltration. The enumeration and typing of inflammatory infiltrate were

840 assessed through immunohistochemical stains (see below). Immune scoring was done by two

841 blinded individuals evaluating at least 10 high powered fields per section for pancreas, lung, liver,

842 heart, thyroid, kidney, salivary, gut (colon and stomach), and gonad tissues; for small tissues

843 (lacrimal glands and eye) 5 high powered fields were evaluated. Images were acquired on an

844 Olympus BX50 microscope using Olympus CellScans Standard software. Images were 845 brightened uniformly for publication in Photoshop. 
848 For immunohistochemistry (IHC), 4mm FFPE tissue sections were deparaffinized, rehydrated, 849 and subjected to heat-induced antigen retrieval $(0.01 \mathrm{~mol} / \mathrm{L}$ citrate, $\mathrm{pH} 6.0)$ followed by treatment 850 with $3 \% \mathrm{H}_{2} \mathrm{O}_{2}$ for 10 minutes to block endogenous peroxidase activity. Sections were incubated 851 overnight at $4^{\circ} \mathrm{C}$ with primary antibodies against mouse IL17A (Abcam, ab91649, 1:50 dil), CD3 852 (DAKO, clone A0542, 1:100 dil), F4/80 (BioRad, clone MAC497G, 1:200 dil), B220 (BD, clone 853 RA3-6B2, 1:50 dil), IL-17RC (Bioss, catalog BS-2607R, polyclonal, 1:300 dil). Sections were then 854 stained with appropriate secondary antibodies and antigen detection done with 3,30855 diaminobenzidine. Sections were counterstained with hematoxylin, dehydrated, and mounted. 856 Appropriate positive and negative controls were used for all stains. Brightness of all images was 857 increased by 50 in Adobe Photoshop. Positively stained leukocytes for CD3, B220, F4/80, or IL858 17A were counted in 10 representative high-power fields (hpf, total 400x magnification) for each 859 tissue section. Two independent observers scored each section and the results were pooled with 860 rare disagreements resolved by a third evaluator.

863 Mouse anti-thyroglobulin antibodies were measured via ELISA as previously reported ${ }^{27}$. Briefly, 86496 -well plates were coated with $50 \mathrm{uL}$ of $1.5 \mathrm{mg} / \mathrm{mL}$ purified mouse thyroglobulin (mTg, generously 865 provided by the Rapoport lab) in Coating Buffer (30mM Na2CO3, 70mM NaHCO3, 6mM NaN3, $866 \mathrm{pH}$ 9.3) covered and incubated at $4^{\circ} \mathrm{C}$ overnight. The following day, plates were washed twice 867 with $100 \mathrm{uL}$ per well Tris/NaCl $(200 \mathrm{mM}$ Tris base, $188 \mathrm{mM} \mathrm{NaCl}, \mathrm{pH} 7.4)$, once with $\mathrm{Tris} / \mathrm{NaCl}$ 868 Tween (Tris/NaCl, 500uL/L Tween 20), and once more with Tris/NaCl. Plates were blocked with 869 100uL/well Blocking Buffer (5\% w/v BSA, 150mM NaCl) for twenty minutes at room temperature $870(\mathrm{RT})$ then washed again as above. Sera samples were diluted 1:100 in PBS and added to wells 871 in triplicate. Plates were covered and allowed to incubate RT 1.5 hours. Plates were washed four 
872 times with $100 \mathrm{uL} /$ well of Tris/NaCl Tween. Anti-mouse IgG1-HRP was diluted 1:2000 in

873 Secondary Antibody Buffer (1\% w/v BSA, 150mM NaCl) and was added to plate at $100 \mathrm{uL} /$ well.

874 Plate was covered and allowed to incubate RT one hour, then washed four times with 100uL/well

875 Tris/NaCl Tween. ELISA was read by adding 100uL/well TMB substrate (Thermo Scientific, 876 N301), allowing 10 minutes to develop in dark, and read on a spectrophotometer at OD 605nM.

878 Thyroid peroxidase antibody

879 Mouse thyroid peroxidase antibody (TPO) antibody levels were detected using mouse sera 880 binding on $\mathrm{CHO}-\mathrm{mTPO}$ cells, followed by flow cytometry as previously described ${ }^{27}$. Briefly, 881 experimental mouse sera were incubated with a single cell suspension of mouse CHO-mTPO 882 cells at a 1:50 dilution in Buffer A ( $2 \%$ FBS in PBS with $10 \mathrm{mM}$ HEPES) for 45 minutes at $4^{\circ} \mathrm{C}$, 883 then washed twice with Buffer A. For detection of immunoglobulin binding, samples were then 884 incubated with goat anti-mouse IgG FITC secondary antibody at $10 \mathrm{ug} / \mathrm{mL}$ for 30 minutes at $4^{\circ} \mathrm{C}$ 885 and then washed twice with Buffer A. Finally, mean fluorescence intensity of FITC was measured 886 for live, single CHO-cells using an Attune NxT 6 cytometer (ThermoFisher).

Flow Cytometry

889 Immediately after euthanasia and perfusion with sterile PBS, fresh tissues were dissociated for 890 analysis of immune infiltrates by flow cytometry. Thyroid glands were dissected away from 891 surrounding trachea and lymphoid tissue, digested in collagenase IV $(1 \mathrm{mg} / \mathrm{mL}$ in $2 \%$ FBS in PBS) 892 at $37^{\circ} \mathrm{C}$ for 20 minutes, then mechanically dissociated by passage through a 40 um filter. Spleen 893 cells were isolated by mechanical dissociation and passage through a 40um filter. To assess 894 intracellular cytokines, cells were incubated in complete RPMI media with 50uM 2ME for four 895 hours with ionomycin $(1 \mathrm{ug} / \mathrm{mL})$ and PMA $(50 \mathrm{ng} / \mathrm{mL})$ in the presence of Brefeldin A prior to 896 staining. For staining, single cell suspensions were resuspended in FACS buffer (0.5mM EDTA, $8972 \%$ FBS in PBS) at $10^{6}$ cells $/ \mathrm{mL}$ and stained with fluorescence conjugated antibodies. For 
898 intracellular staining, after surface staining, cells were fixed and permeabilized using cytoplasmic 899 fixation and permeabilization kit (BD, for cytokines IL-17A and IFN $\gamma$ ) or FoxP3 transcription factor 900 kit (eBioscience, for ROR $\gamma \mathrm{t}$ ), per protocol instructions, with 30 min fixation at $4^{\circ} \mathrm{C}$. Viability dye 901 DAPI was added prior to analysis where indicated. Cells were then washed twice in FACS buffer

902 and analyzed by flow cytometry on an Attune NxT 6 cytometer (ThermoFisher). Antibodies used 903 are shown in Supplemental Methods. Cell counts are shown as relative frequency of live, gated 904 single cells unless otherwise noted. For determination of infiltrating cells per thyroid lobe, whole 905 perfused thyroid specimens were dissociated to single cell suspensions and the entire specimen 906 stained for flow cytometry and a fixed volume of the specimen was evaluated by flow cytometry 907 to allow back calculation for estimate of absolute cell count (e.g. 100ul of 200ul total sample 908 volume run yields a $2 x$ multiplier for cell count).

$910 \quad$ Neutralizing antibody studies in ICI-treated mice

911 Groups of 4-6 wk old NOD mice were randomized to treatment with anti-mouse PD-1 (clone 912 RPM1-14) plus anti-mouse CTLA-4 (9D9) antibodies or isotype controls (2A3 plus MPC-11) at $91310 \mathrm{mg} / \mathrm{kg} /$ dose twice weekly by i.p. injection. Additionally, 10 days after start of ICl or isotype 914 therapy, mice were further randomized to receive neutralizing antibody to IL-17A (clone 17F3), 915 TNFa (clone XT3.11), combination or vehicle alone (PBS).

917 Tumor model studies

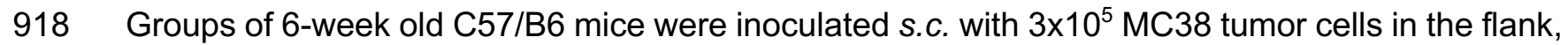
919 as described previously ${ }^{74}$, and randomized into groups $(n=6)$ for treatment when tumor volumes 920 reached $40-80 \mathrm{~mm}^{3}$. Groups of mice received no treatment (PBS vehicle only), combination ICl 921 [anti-PD-1 (clone RPM1-14) + anti-CTLA-4 (clone 9D9) at 10mg/kg/dose], or combination ICI with 922 a neutralizing antibody to mouse IL-17A (clone 173F, 0.5mg/dose) or isotype control (catalog 
923 BE0088). Reagents were given by i.p. injection on the ipsilateral side to the tumor. Mouse tumor

924 volumes were measured every 2 days by caliper and mice were sacrificed when tumor volumes

925 reached $2 \mathrm{~cm}$ in diameter or when animal morbidity mandated sacrifice under institutional vivarium

926 protocols.

928 Stimulation of human thyroid cell line NThy-Ori-3.1

929 To determine the effects on antigen presentation and cytokine production of IL-17A and TNFa on

930 the human thyroid cell line NTHy-Ori 3.1, an in vitro test of cytokine exposure was used to evaluate

931 RNA for gene expression changes. The $1.0 \times 10^{5}$ Thy Ori 3.1 cells were plated in $1 \mathrm{~mL}$ of $10 \%$

932 RPMI in a 24-well plate. Once the cells were adherent, $50 \mathrm{ng} / \mathrm{mL}$ of each cytokine or vehicle (PBS,

933 IL-17A, TNF $\alpha$, or IL-17A+TNF $\alpha$ ) was added per well and were allowed to incubate at $37^{\circ} \mathrm{C}$. Each

934 condition had four biological replicates. After the eight hour stimulation, cells were used for RNA

935 extraction and quantitative reverse transcriptase PCR.

936

937 Quantitative reverse transcriptase PCR (qRT-PCR)

938 For RNA extraction, human NThy-Ori-3.1 cells were washed twice with PBS, then lysed directly

939 in tissue culture wells. For mice experiments, resected mouse thyroid lobes were snap frozen in

940 liquid nitrogen and stored at $-80^{\circ} \mathrm{C}$. Multiple thyroid lobes were homogenized in RNA Lysis Buffer

941 (Zymo Research) using the Fisherbrand 150 Handheld Homogenizer (15-340-167). RNA

942 extractions were performed using Quick-RNA Microprep kit (Zymo Research, R1051) per

943 manufacturer's instructions. RNA yield was quantified by nanodrop. RNA was converted into

944 cDNA using High-Capacity cDNA Reverse Transcription Kit with RNAse Inhibitor and MultiScribe

945 Reverse Transcriptase (Applied Biosystems, 4374966) following manufacturer's instructions.

946 About $750 \mathrm{ng} / \mathrm{uL}$ of RNA was used for cDNA synthesis per reaction. Quantitative PCR was

947 performed using Taqman Fast Advanced PCR Master Mix (Applied Biosystems, 4444557) and

948 Taqman probes for genes of interest (Supplemental Methods), with three technical replicates 
per gene, per condition. PCR cycles were run using the QuantStudio 6 Pro PCR machine (Applied

950 Biosystems) with the standard cycle parameters. Design and Analysis QuantStudio 6/7 Pro

951 Systems Software (Thermo Fisher Scientific, Version 2.5.0) was used to identify amplification of

952 genes and calculate fold change from Cq values. In this context, fold change was the expression

953 ratio of the gene of interest to the housekeeping gene, GAPDH. For further analysis of NThy-Ori-

9543.1 antigen presentation and cytokine production, human 293T cells were also used for qRT-PCR

955 to compare upregulated gene expression.

957 Patients

958 Patients were prospectively enrolled from two academic medical centers (UCLA Health, USC

959 Keck Medical Center) under IRB approved protocols (19-000032, 19-001708, HS-19-00715).

960 Peripheral blood specimens were collected from adult (age $>18$ years) patients under several

961 categories: ICl-treated cancer patients ( $n=20$, including 18 with thyroid or other IrAE or no IrAE),

962 Hashimoto's thyroiditis (HT, $n=13)$, or healthy control $(n=6)$ with no evidence of thyroid disease.

963 Exclusion criteria included pregnancy, history of thyroid surgery, radioactive iodine therapy, or

964 thyroid cancer, immune modifying conditions not including solid malignancy (e.g. bone marrow 965 transplantation, leukemia or lymphoma, known genetic or acquired immunodeficiency, or immune 966 modifying medications at the time of specimen collection, excluding physiologic steroids). ICl967 treated subjects must have received an FDA-approved ICI within the past 1 month. ICI treatment 968 included combination anti-CTLA-4 + anti-PD-1 in 5 subjects, single agent anti-PD-1/L1 in 8, and 969 sequential in 7 (anti-CTLA-4 + anti-PD1, then anti-PD-1 in 6 subjects and anti-PD-1, followed by 970 combination in 1 subject). Cancer types were as follows: melanoma $(n=9)$, renal cell $(n=5)$, breast $971(n=2)$, lung squamous cell $(n=1)$, sacroma $(n=1)$, neuroendocrine $(n=1)$ hepatocellular $(n=1)$. HT 972 patients had hypothyroidism (elevated TSH and low FT4, and/or requirement for thyroid hormone 973 replacement) and evidence of thyroid autoimmunity (e.g. thyroid auto-antibody presence: anti974 TPO or anti-Tg); imaging findings if available were consistent with HT. Controls without thyroid 
975 disease included subjects with normal thyroid hormone status (TSH and FT4), absence of anti-

976 TPO and anti-Tg antibodies, and no history of thyroid disease; subjects may have a benign

977 nodule. Peripheral blood mononuclear cells were isolated from blood by density gradient 978 centrifugation (Ficoll).

979

$980 \quad$ Single cell RNA sequencing

981 For mouse scRNAseq studies, $\mathrm{CD}^{4} 5^{+}$infiltrating cells were isolated from fresh thyroid tissue. To 982 reduce contamination from circulating immune cells in the blood, animals were perfused with 983 normal saline prior to tissue collection. Thyroid tissue was enzymatically and mechanically 984 dissociated, then single cell suspensions stained with fluorescence-conjugated antibodies to 985 CD45, CD11b, CD3, CD4, CD8, and CD19 and viability dye DAPI. Specimens with and potential 986 cervical LN or thymus contamination were excluded by detection of $>20 \%$ double positive CD4 ${ }^{+}$ $987 \mathrm{CD}^{+} \mathrm{T}$ cells ${ }^{75} \cdot 10,000$ cells were submitted for 10x single cell transcriptomic sequencing. Library 988 construction and sequencing was done by the UCLA Technology Center for Genomics and 989 Bioinformatics (TCGB) core facility.

990

991 Data processing of scRNA-seq libraries

992 After sequencing, the scRNA-seq reads were aligned to the mm10 (mouse) reference genome 993 and quantified using the 10x Genomics cellranger count software. Filtered output matrices that 994 contained only barcodes with unique molecular identifier (UMI) counts that passed the threshold 995 for cell detection were used for downstream analysis.

997 Normalization, Principal Component analysis, and UMAP Clustering

998 Downstream analysis was done with Seurat v3 ${ }^{45}$. Only cells with a mitochondrial gene percentage 999 less than $30 \%$ and 200 features were included in downstream analysis. Scores for S and G2/M 1000 cell cycle phases were assigned using the Seurat CellCycleScoring function following the 
1001 standard Seurat pipeline ${ }^{76}$. UMI counts were log normalized, and the top 2000 variable genes 1002 were determined using the variance-stabilizing transformation (vst) method. All genes were 1003 scaled and centered using the ScaleData function, and principal component analysis (PCA) was 1004 run for the data using the predetermined variable genes. To group cells into clusters, a K-nearest 1005 neighbors graph function (implemented in the Seurat package), followed by a modularity1006 optimizing function using the Louvain algorithm was used. For the cluster, 30 PC dimensions were 1007 included and the resolution parameter was set to 0.4. Cell-type clusters were visualized using 1008 uniform manifold approximation and projection (UMAP) to reduce dimensionality and allow for the 1009 cells to be visualized on a 2-D plot.

1010

1011 Differential Expression and Marker Gene Identification

1012 The Seurat findMarkers function was used to generate the top upregulated genes for each cluster 1013 using a Wilcoxon Rank Sum Test to identify differentially expressed genes across clusters. Marker 1014 genes were filtered by a minimum of detectable expression in $25 \%$ of the cells in the target group 1015 and minimum log2 fold change of 0.25 . The markers generated by these functions were compared 1016 to markers for known cell types to assign identities to the different clusters. Differentially 1017 expressed genes across conditions were identified using the same function and parameters.

1019 Integration and Subsetting of Datasets

1020 For analysis of different immune cell subsets ( $T$ cell, Rorc ${ }^{+}$and B/myeloid cells), variable features 1021 were recomputed for each subset and downstream analyses including scaling, PCA, and UMAP 1022 were rerun. Initial cell clustering for CD45 ${ }^{+}$immune cells identified a cluster of likely cervical 1023 thymus contamination origin (double positive Cd4 and Cd8a-expressing cells by correlation 1024 analysis, with co-expression of Cd24a, Rag2, Rag1, and Dntt) as previously reported in mouse 1025 neck tissue ${ }^{75}$. To remove potential thymic contamination from subsequent Cd3 and Rorc 1026 subclustering, cells expressing Rag1 at an expression level greater than 1 were removed. For 
1027 Cd3 subclustering, $C d 3^{+}$subsets were manually selected from $C D 45^{+}$UMAP and reclustered. 1028 Similarly, B cell, myeloid and APC subclustering from mouse CD $45^{+}$cells was done similarly. For 1029 the Rorc $^{+}$subclustering in mouse dataset, all cells from the T-cell subset expressing Rorc at an 1030 expression level greater than 1 were selected for analysis.

1032 We used the CellChat $\mathrm{R}$ package (version 0.5.5) to makes inferences about potential cell-cell 1033 interactions using the gene expression of murine receptors and ligands in the CellChatDB, a 1034 database curated by Jin et al. ${ }^{77}$ containing genes from peer-reviewed literature and the Kyoto 1035 Encyclopedia of Genes and Genomes (KEGG). CellChat performs a comprehensive analysis of 1036 signaling gene expression; its database considers multiple complexes' subunits, which must all 1037 be expressed for a signaling pathway to be considered significant. The same requirements were 1038 also applied on cofactors and mediators of cell communication. These curated signaling genes 1039 are grouped into biologically relevant pathways such as CXCL and CCL (genes comprising each 1040 pathway shown in Extended Data Table 2).

1042 Transcriptomic data from thyroid-infiltrating $\mathrm{Cd} 45^{+}$immune cells from ICl-treated mice were 1043 imported from the Seurat analysis pipeline which allowed CellChat to make use of the manually 1044 assigned cluster labels. Our analysis closely followed the example created by Jin et al. ${ }^{77}$ 1045 (https://github.com/sqjin/CellChat). CellChat requires the data to be sub-sampled using geometric 1046 sketching to reduce the computational workload using the subsetData function ${ }^{78}$. Comparison of 1047 expression of genes from CellChatDB across cell clusters was performed with the 1048 identifyOverExpressedGenes and identifyOverExpressedlnteractions functions using a Wilcoxon 1049 rank sum test using the default significance level of 0.05 . Communication probability (or strength 1050 of interaction) was calculated using an equation described in Jin et al. ${ }^{77}$ based on the law of mass 1051 action using the function computeCommunProb. Average gene expression was calculated using 1052 the default trimean approach. Signaling pathways were determined to be significantly expressed 
1053 using a permutation test using the default number of permutations $(M=100)$ and default 1054 significance threshold $(p$-value $<0.05)$. Signaling pathways were filtered to remove those 1055 expressed in fewer than 10 cells in a single cluster using the filterCommunication function. 1056 Signaling probabilities for each pathway were generated by analyzing the average expression of 1057 each pathway's receptors and ligands computed by earlier analysis using the 1058 computeCommunProbPathway function. The source, recipient, and mediating cell groups were 1059 determined by analyzing the average pathway expression by each cell cluster using the 1060 aggregateNet function and the netAnalysis_computeCentrality function. These analyses were 1061 visualized using CellChat's circle and hierarchy plot and heatmap functions.

1062

1063 Statistics

1064 Data were analyzed with GraphPad Prism v9. Descriptive statistics shown are mean + SEM for 1065 continuous variables. Differences in the mean frequency of immune cells or gene expression for 1066 qRT-PCR between two groups were evaluated by two-sided t test with Welch correction for 1067 potential differences in variance among groups and correction for multiple comparisons by the 1068 Holm-Sidak method. For B6 mice, differences in organ immune infiltrate score, thyroid 1069 autoantibodies (TPO MFI and Tg ELISA OD) were compared between groups by two-tailed, 1070 unpaired $t$ test with Welch correction, without assumption of equal s.d. Differences in the mean 1071 frequency of immune cells, organ immune infiltrate score, thyroid autoantibodies (TPO MFI and 1072 Tg ELISA OD) among groups were evaluated by Brown-Forsythe ANOVA with Welch correction 1073 for potential differences in variance among groups, followed by multiple comparisons between 1074 groups with Dunnett correction for multiple comparisons (two-tailed comparisons). Where 1075 appropriate, adjusted $p$ values are shown. Significance was set at an alpha $=0.05$. Samples sizes 1076 for each group or condition are shown. 
1079 Data associated with figures are available from the corresponding author upon reasonable 1080 request. The datasets for single cell sequencing generated during and analyzed during the current 1081 study will be available in the GEO repository (https://www.ncbi.nlm.nih.gov/geo/).

1082

1083 Methods References

108474 Moreno, B. H. et al. Response to programmed cell death-1 blockade in a murine 1085 1086 melanoma syngeneic model requires costimulation, CD4, and CD8 T cells. Cancer Immunol. Res. 4, 845-857 (2016).

1087 75. Terszowski, G. et al. Evidence for a functional second thymus in mice. Science 312, 284$1088287(2006)$.

1089 76. Tirosh, I. et al. Dissecting the multicellular ecosystem of metastatic melanoma by single1090 cell RNA-seq. Science (80-. ). 352, 189-196 (2016).

1091 77. Jin, S. et al. Inference and analysis of cell-cell communication using CellChat. bioRxiv 11092 20 (2020) doi:10.1101/2020.07.21.214387.

78. Hie, B., Cho, H., DeMeo, B., Bryson, B. \& Berger, B. Geometric Sketching Compactly Summarizes the Single-Cell Transcriptomic Landscape. Cell Syst. 8, 483-493.e7 (2019).

\section{Acknowledgements}

1097 This work was supported by funding from the American Thyroid Association 1098 (THYROIDGRANT2020-0000000169, M.G.L.), UCLA Specialty Training and Advanced Research 1099 (STAR) program (M.G.L.), Parker Institute for Cancer Immunotherapy (M.A.S.), and UCLA core 1100 facilities [Broad Stem Cell Research Center FACS core, Translational pathology core laboratory 1101 (TPCL), and Technology Center for Genomics and Bioinformatics (TCGB)]. The authors would 1102 like to acknowledge Drs. Sandra McLachlan and Basil Rapoport for CHO-mTPO cell line and 1103 methods for thyroid autoantibody detection. 
1106 M.G.L., W.H., T.E.A., A.D., and M.A.S. conceived the study and designed the analysis.

1107 M.G.L., A.Y.P., T.E.A., M.S.P., N.Y., M.I.C., A.T.H., and L.G. collected the data, including 1108 mouse experiments with $\mathrm{ICI}$ treatment, patient specimens, flow cytometry, qRT-PCR, 1109 immunohistochemistry, and tumor model studies. W.H., A.R., A.D., H.C., and M.A. S. 1110 contributed data and analysis tools. M.G.L., A.Y.P., M.S.P., N.Y., A.T.H., E.C.M., E.D.R., 1111 and H.C. performed the analysis. M.G.L., A.Y.P., W.H., T.E.A., M.A.S., A.T.H., H.C., N.Y., 1112 E.C.M., A.D., A.R., and M.A.S. wrote or edited the paper. All authors reviewed the 1113 manuscript.

\section{Competing interests}

1116 A.R. has received honoraria from consulting with Amgen, Bristol-Myers Squibb, Chugai, 1117 Genentech, Merck, Novartis, Roche, Sanofi and Vedanta, is or has been a member of the 1118 scientific advisory board and holds stock in Advaxis, Appia, Apricity, Arcus, Compugen, CytomX, 1119 Highlight, ImaginAb, Isoplexis, Kalthera, Kite-Gilead, Merus, PACT Pharma, Pluto, RAPT, 1120 Rgenix, Synthekine and Tango, has received research funding from Agilent and from Bristol1121 Myers Squibb through Stand Up to Cancer (SU2C), and patent royalties from Arsenal Bio. All 1122 other authors have declared no conflicts of interest.

\section{Additional information}

1125 Extended data is available for this paper.

1126 Supplementary information is available for this paper.

1127 Correspondence and materials requests should be directed to Dr. Melissa Lechner, Division of 1128 Endocrinology, Diabetes, and Metabolism, UCLA, 10833 Le Conte Ave, CHS 57-145, Los

1129 Angeles, CA, 90095. Email: MLechner@mednet.ucla.edu; Phone: 310-794-3237. 
Extended Data Tables

1133 Extended Data Table 1. Top gene lists for scRNAseq data.

1134 Extended Data Table 2. Ligand-Receptor interaction pathway genes in CellChat analyses.

\section{Extended Data Figure Legends}

1137 Extended Data Fig. 1. Flow cytometry analysis of thyroid-infiltrating immune cells in anti-

1138 PD-1 + anti-CTLA-4 (Dual ICI) treated B6 mice. Mice were euthanized after 4 weeks of ICl or 1139 isotype treatment, perfused with saline, and then fresh thyroid tissues dissociated into single cell 1140 suspensions. Cells were stained with fluorescent antibodies and analyzed by flow cytometry, with 1141 estimation of cells/thyroid lobe for each animal. Each point represents an individual mouse ( $n=8$

1142 isotype and $n=8$ Dual $(\mathrm{Cl})$. Mean frequencies of cell populations shown between groups were 1143 compared by two-tailed, unpaired t test with Welch correction, assuming unequal s.d., and Holm1144 Sidak method correction for multiple comparisons, with $p<0.05$ considered significant. The $p$ 1145 values for all comparisons were not significant.

1148 mice. Representative H\&E micrographs showing autoimmune tissue infiltrates in Dual ICI vs. 1149 isotype treated mice (original mag. 100x-400x). Shown are isotype treated mice tissues (left) and 1150 Dual ICl-treated mice tissues (right) for organs noted to have infiltrates in multiple animals. Arrows 1151 indicate areas of autoimmune infiltration.

1153 Extended Data Fig. 3. Flow cytometry analysis of peripheral immune changes in ICl-treated 1154 NOD mice. Frequency of putative natural killer (NK, NKp46 ${ }^{+}$CD3-), NKT $\left(\mathrm{CD}^{+}{ }^{+} \mathrm{NKp} 46^{+}\right), \mathrm{B}^{-}$

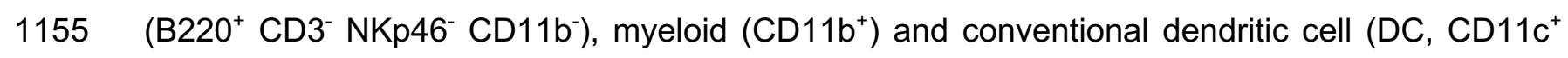


$\left.1156 \mathrm{CD} 11 \mathrm{~b}^{\text {low }}\right)$ as a percent of splenocytes in isotype $(n=7)$ vs. anti-PD-1 + anti-CTLA-4 (Dual ICI,

$1157 n=8$ ) mice after 4 weeks of treatment. Differences in immune populations in spleen were 1158 compared by two-tailed, unpaired t test with Welch correction, assuming unequal s.d., and Holm1159 Sidak method correction for multiple comparisons. The adjusted $p$ values are as follows: NK cell $1160 \quad 0.004$, NKT cell 0.002 , B cell 0.12, CD11 $^{+}$myeloid cell 0.3 , and DC 0.16 .

1162 Extended Data Fig. 4. Additional scRNAseq analysis of thyroid infiltrating immune cells 1163 from ICl-treated NOD mice.

1164 a, Heatmap showing top 10 variable genes expressed in cell clusters for CD45 ${ }^{+}$thyroid-infiltrating 1165 immune cells from ICl-treated NOD mice. $b$, Feature plots showing expression of immune cell 1166 population-associated genes $\mathrm{CD}^{4} 5^{+}$thyroid-infiltrating immune cells. $c$, Heatmap showing top 10 1167 variable genes expressed in cell clusters for sub-clustered $C d 3^{+}$cells. $d$, Violin plots for type 1 1168 immunity (top left, Tbx21, Ifng), T cell activation (bottom left, Cd69 and Cd44), and cytotoxicity1169 associated (right, Prf1, Gzmk, Fasl, and Nkg7) genes in cell clusters for sub-clustered Cd3 cells. 1170 e, Heatmap showing top 20 variable genes expressed in cell clusters for sub clustered Rorc $^{+}$cells.

1174 a, Subcluster of myeloid and B cells from CD45 ${ }^{+}$thyroid infiltrating cells in ICl-treated mice. UMAP 1175 shows 7 distinct clusters. $b$, Heatmap of top 10 genes in subclustered cells. $c$, Feature and violin 1176 plots for expression of inflammatory cytokines including Tnf, $/ 1$, and Tgfb1. d, Intrathyroidal gene 1177 expression in ICl-treated vs. isotype mice measured by qRT-PCR ( $n=12$ animals group for all 1178 genes except $n=4$ for $/ / 1 b$; thyroid tissue pooled and run in 3 experiments; qRT-PCR reactions in 1179 triplicate). Relative gene expression shown as mean fold change \pm SEM for ICl-treated fold 1180 change over isotype. Comparison by two-tailed, unpaired t test with Welch correction, assuming 1181 unequal s.d., and Holm-Sidak method correction for multiple comparisons. Adjusted P values are 
$1182<0.0001$ for Tnf, II6, and Tgfb1; one pooled sample evaluated for I/1b so statistical significance

1183 was not tested. e, Macrophage and dendritic cell clusters showed likely signaling interactions via

$1184 \mathrm{CXCL}$ pathways to effector CD4 and CD8, $\gamma \delta \mathrm{T}$, and NK/NKT cells, as predicted by ligand-receptor 1185 interactions in transcriptomic data using CellChat.

1187 Extended Data Fig. 6. Potential action of IL-17A on thyroid epithelial cells.

1188 a, IHC staining for IL-17 receptor C (IL-17RC) in thyroid tissue of Dual ICl-treated mice (top left).

1189 Murine stomach tissue stained for IL-17RC positive control (bottom left) and secondary only 1190 controls (right) shown for comparison (representative sections, original mag. 400x). $b$, Relative 1191 gene expression of IL-17RC (II17rc) measured by qRT-PCR in human NThyOri-3.1 cells treated 1192 with vehicle alone ( $(n=3)$, recombinant human IL-17A $(n=3), \operatorname{TNF} \alpha(n=3)$, or both $(n=3)$ for 8 hours. 1193 Gene expression normalized to housekeeping gene and shown relative to human 293T cells 1194 (n=4). Comparison of NThy-Ori-3.1 to 293T cells by two-tailed, unpaired t test with Welch 1195 correction, assuming unequal s.d., with $p$ value of 0.04 .

1196

1197 Extended Data Fig. 7. Gating strategy and representative dot plot for human peripheral 1198 blood mononuclear cell staining for T cell populations and interleukin (IL)-17A. 

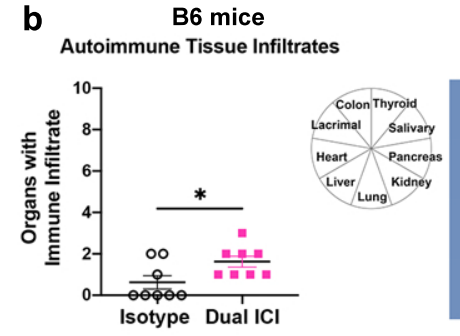

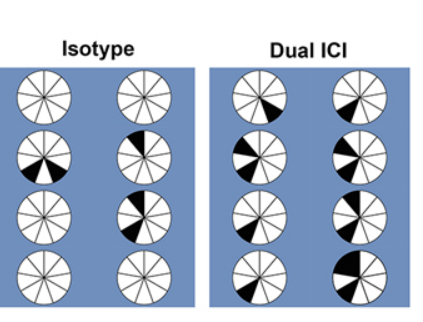

C

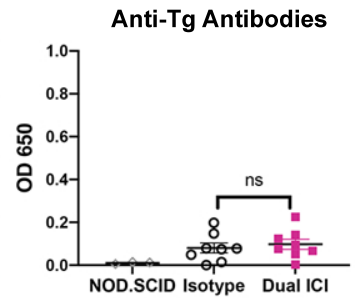

B6 mice

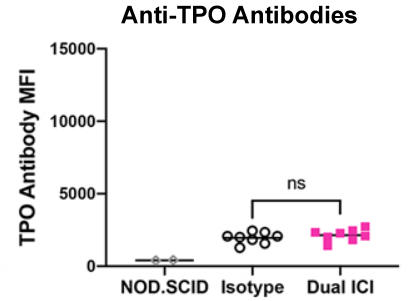

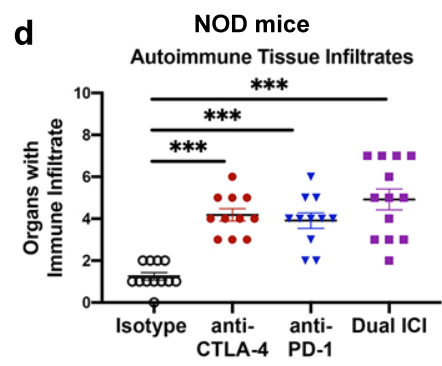

$\mathbf{e}$

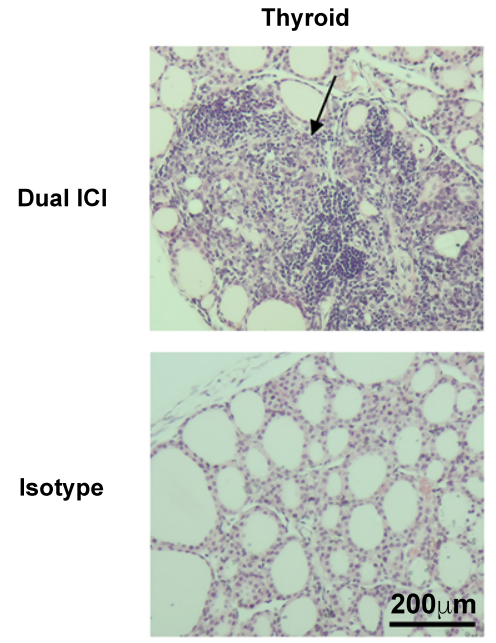

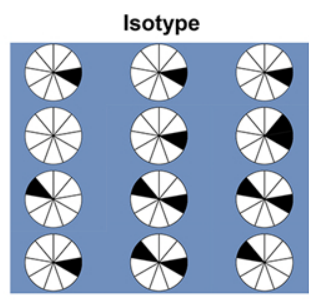
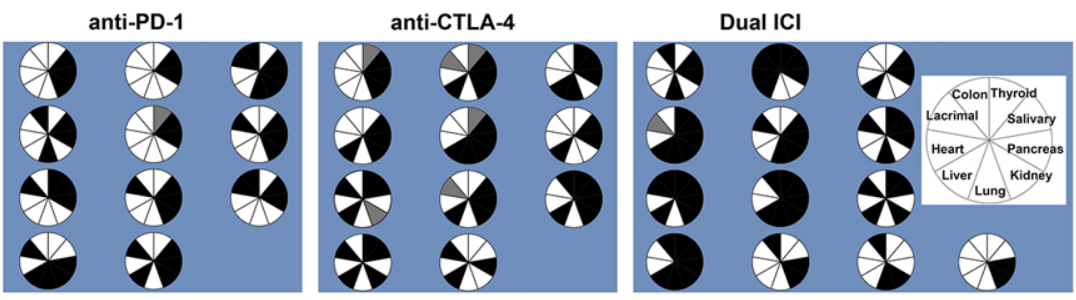

$\mathbf{f}$

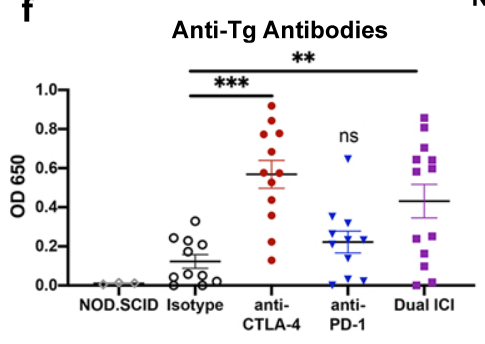

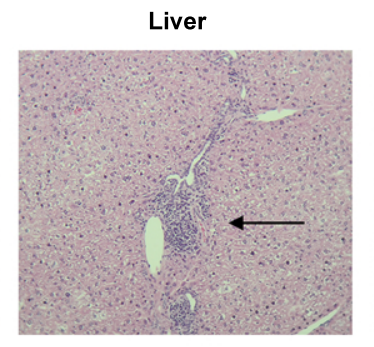

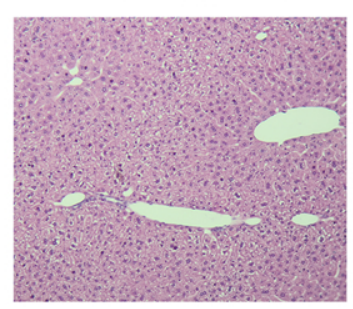

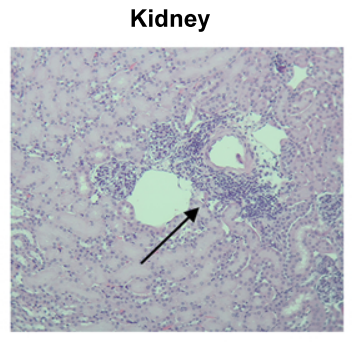

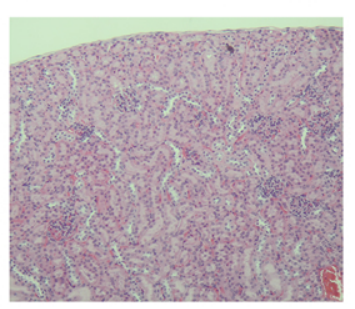

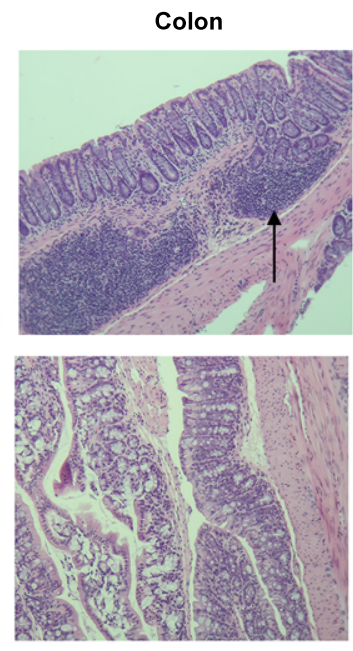

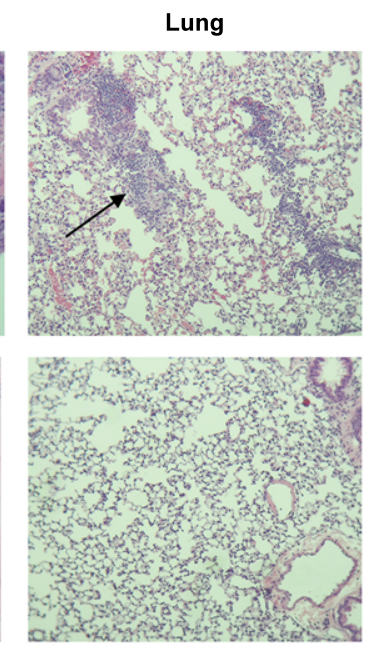

NOD mice

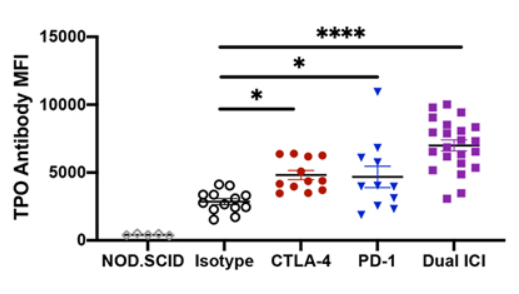

g

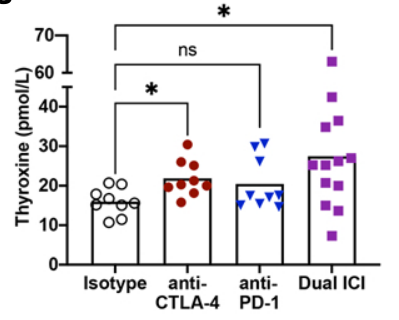

Figure 1. ICl therapy induces multi-system autoimmune infiltrates (IrAE) in B6 and NOD mice.

a, Schematic of ICl drug treatment. b. Comparison of autoimmune organ infiltration after 4 weeks of ICI vs. isotype treatment in C57/B6 (B6) mice ( $n=8$ isotype, $n=8$ Dual ICl); Pie charts (right) show tissue infiltrate for each mouse; black $=$ immune infiltrate, white $=$ no infiltrate, gray $=$ no data. c, Quantification of antithyroid autoantibodies in B6 mice after 4 weeks of isotype $(n=8)$ or Dual $\mathrm{ICI}(n=8)$ treatment. $d$, Comparison of autoimmune organ infiltration after 8 weeks of $\mathrm{ICl}$ treatment in NOD mice ( $n=12$ isotype, $\mathrm{n}=11$ anti-CTLA$4, n=11$ anti-PD-1, and $n=13$ Dual ICI). Pie charts show tissue infiltrate for each mouse, as in $\mathbf{b}$ above. e, Representative H\&E micrographs of autoimmune tissue infiltrates in Dual ICl vs. isotype treated mice (original mag. 100x). f, Quantification of anti-thyroid autoantibodies in NOD mice after 8 weeks of treatment ( $n=10$ isotype anti-Tg or 13 for anti-TPO, $n=12$ anti-CTLA-4, $n=11$ anti-PD-1, and $n=13$ Dual ICl for anti-Tg or 22 for anti-TPO). g, Free thyroxine serum levels in ICl-treated NOD mice after 8 weeks of treatment. Data are mean \pm SEM. ${ }^{*} p<0.05,{ }^{* *} p<0.01,{ }^{* * *} p<0.001$, unpaired t test, two-tailed (b,c), Brown-Forsythe ANOVA, assuming unequal s.d., followed by Dunnett's multiple comparisons test $(\mathbf{d}, \mathbf{g})$, or one-way ANOVA and Dunnett's multiple comparison test (f). 
Figure 3. Characterization of thyroid-infiltrating immune cells in ICl-treated NOD mice.

a, IHC staining for T cells (CD3), macrophages (F4/80), and B cells (B220) in thyroid tissue from Dual ICl$(n=7,10$, or 9$)$ vs. isotype- $(n=4)$ treated mice [left, representative sections, $400 x$ original mag.; right, cells/hpf]. b, Representative gating strategies and dot plots (left) and relative frequency of CD45+ immune cells in thyroid tissue (right, cells per thyroid lobe) from isotype $(n=11)$ vs. Dual ICl $(n=17)$ treated mice after 4 weeks. c, Relative frequency of immune subpopulations in isotype $\left(n=11\right.$, except $n=16$ for $\mathrm{CD}^{+}$and $\left.\mathrm{B} 220^{+}\right)$vs. Dual ICl-treated $\left(n=17\right.$, except $n=23$ for $\mathrm{CD}^{+}$and $\left.\mathrm{B}^{2} 20^{+}\right)$mice. d, Schematic of tissue processing for single cell RNA sequencing (left). UMAP plot of 4,095 thyroid-infiltrating CD $45^{+}$immune cells from 10 pooled Dual ICl-treated mice, 2 independent experiments (right). Cluster analysis yields 14 distinct clusters comprising CD4, CD8, and $\gamma \delta$ T cells, natural killer (NK)/NKT cells, innate lymphoid cells (ILC), B cells, macrophages, and dendritic (DC)/antigen presenting cells (APC). e, Intrathyroidal gene expression for $\mathrm{Cd} 45$ and $\mathrm{Cd} 3 \mathrm{e}$ in ICl-treated vs. isotype mice measured by qRT-PCR. $\mathbf{f}$, UMAP of CD3-subclustered cells, $n=3,178$ cells. Cluster analysis yields 10 distinct clusters comprising CD4, CD8, and $\gamma \delta$ T cells (left). Feature and violin plots for T cell subset markers for $\delta \gamma(T r d c)$, CD4 (Cd4), and CD8 (Cd8a) (right). g, Violin plots for type 3 immunity (ROR $\gamma$ t-pathway) associated genes Rorc and II23r. h, Intrathyroidal gene expression for Rorc, II23r, and I/17a in ICl-treated vs. isotype mice, by qRT-PCR. i, Comparison of thyroidinfiltrating ROR $\gamma t^{+}$pan-CD3 ${ }^{+}(\alpha \beta$ and $\gamma \delta)$ T cells by flow cytometry in ICl-treated $(n=8) v s$. isotype $(n=6)$ mice. Data are mean \pm SEM. For qRT-PCR, mean fold change \pm SEM, $n=12$ animals/group; thyroid tissue pooled and run in 3 experiments, in triplicate. ${ }^{*} \mathrm{p}<0.05$, ${ }^{* *} \mathrm{p}<0.01$, two-tailed, unpaired $\mathrm{t}$ test with Welch correction, assuming unequal s.d. (a-c,i) and Holm-Sidak method correction for multiple comparisons (e,h). 


\section{Lechner et al. Figure 4}

a

Subclustered on $\mathrm{RORC}^{+}$cells

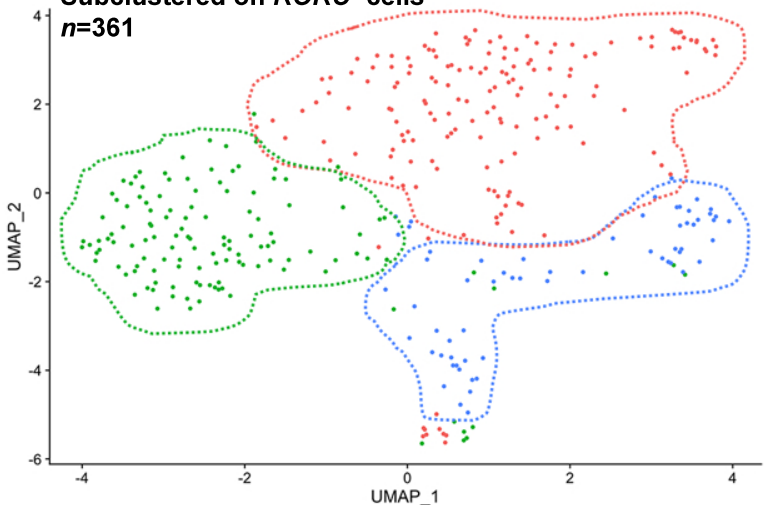

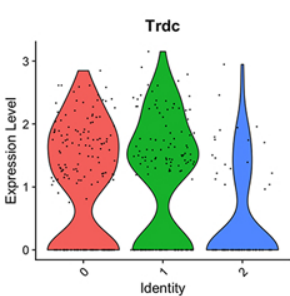
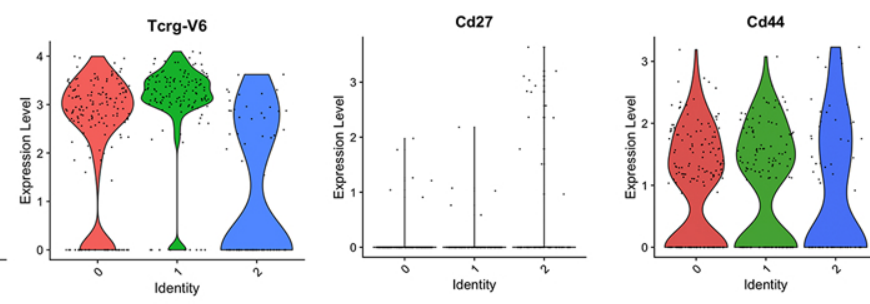

Cd8a
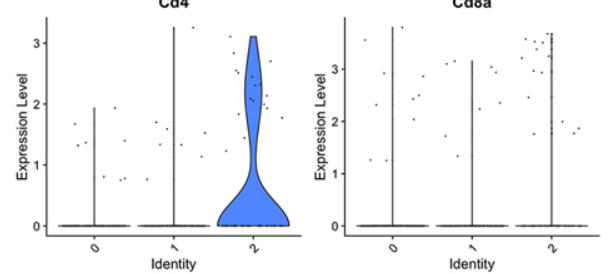

b
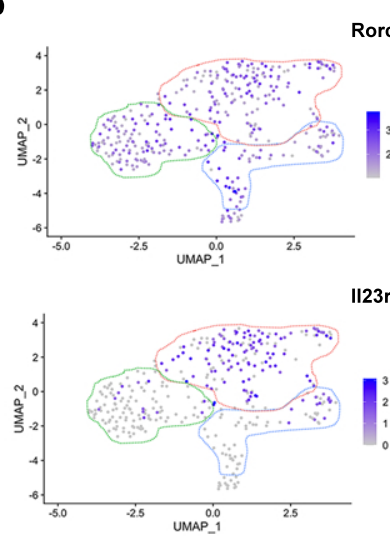

1123
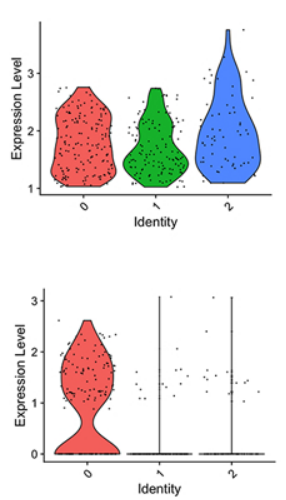

C

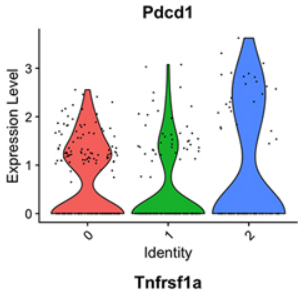

d

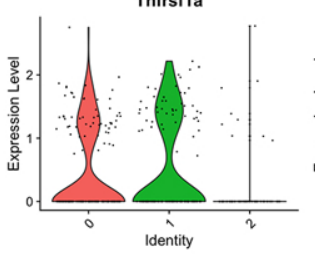

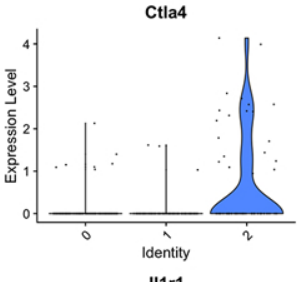

II1 $\mathrm{r} 1$

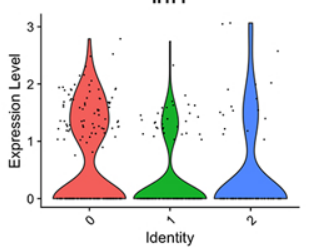

e

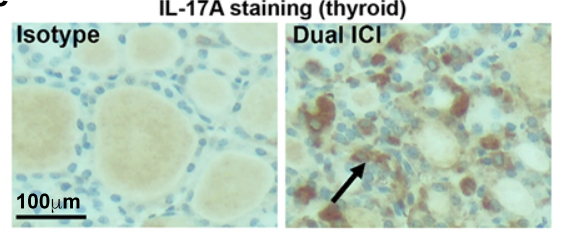

f
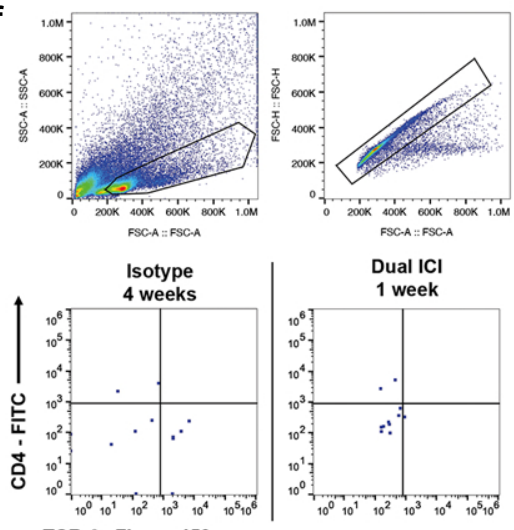

TCR $\gamma \delta$-Fluor e450

h
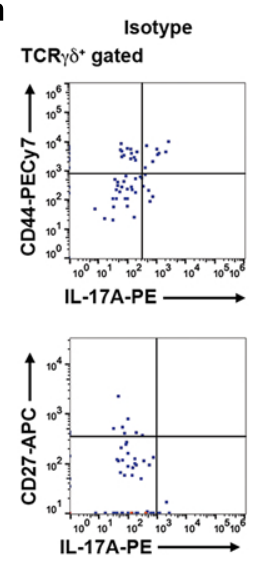

anti-CTLA-4
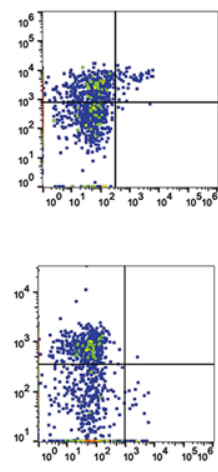

g

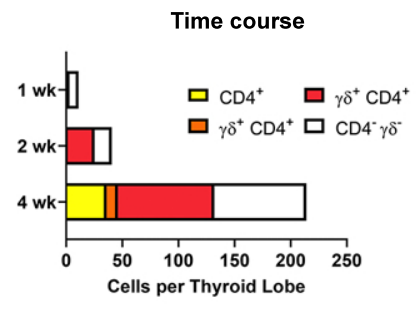

Intrathyroidal IL-17A ${ }^{+}$cells in ICI-treated mice Difference by $\mathrm{ICl}$ regimen

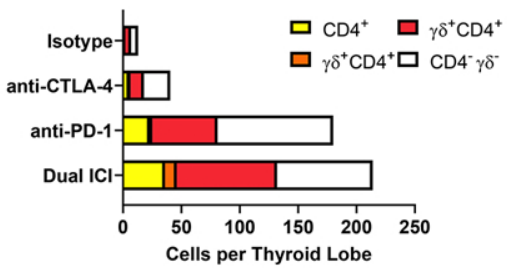

Figure 4. ROR $\mathrm{rt}^{+} \mathrm{IL}-17 \mathrm{~A}^{+} \mathrm{T}$ cells in thyroid immune infiltrates of $\mathrm{ICl}$-treated mice.

a, UMAP of Rorc-subclustered cells, $n=361$ cells. Cluster analysis yields 3 distinct clusters (left) comprised of CD4 and $\gamma \delta$ T cells, as shown by violin plots (right). b, Feature and violin plots for Th17and $\gamma \delta$ T17-associated genes. c-d, Violin plots for checkpoint proteins (c) Pdcd1 and Ctla4 and inflammatory cytokine receptors (d) Tnfrsf1a and I/1r1 on Rorc ${ }^{+}$thyroid-infiltrating T cells. e, IHC staining for IL-17A in thyroid tissue of isotype and Dual ICl-treated mice (representative sections from 3 independent experiments, original mag. 400x). f, Gating strategy and representative dot plots of IL-17A ${ }^{+}$CD4+ (Th17) and $\gamma \delta^{+}(\gamma \delta T 17)$ cells in thyroid immune infiltrates in isotype or Dual ICl-treated mice at $1-4$ weeks $(n=2$ pooled animals each condition). g, Quantification of intrathyroidal $\mathrm{CD}^{+}{ }^{+} \mathrm{IL}_{-} 17 \mathrm{~A}^{+}$and $\mathrm{TCR} \gamma \delta^{+} \mathrm{IL}-17 \mathrm{~A}^{+}$cells from thyroid immune infiltrates from $\mathrm{ICl}$-treated mice at different timepoints of $\mathrm{ICl}$ thyroiditis development. $\mathbf{h}$, Representative gating strategy and flow cytometry dot plots showing $\mathrm{CD}_{4}{ }^{+}$and CD27- phenotype of TCR $\gamma \delta^{+}$IL-17A ${ }^{+}$cells. g-h, for plots shown, pooled thyroid immune infiltrates from isotype $(n=2)$, anti-PD-1 $(n=3)$, anti-CTLA-4 $(n=4)$, and Dual $\mathrm{ICl}(n=2)$ treated mice after 4 weeks, or sooner timepoints $(n=2)$ from Dual ICl treated for Time Course. 
a

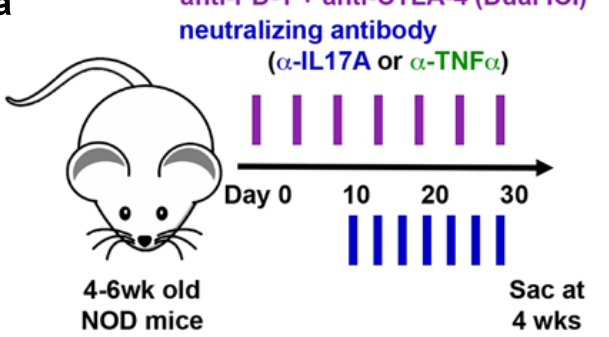

b Autoimmune Organ Infiltrate

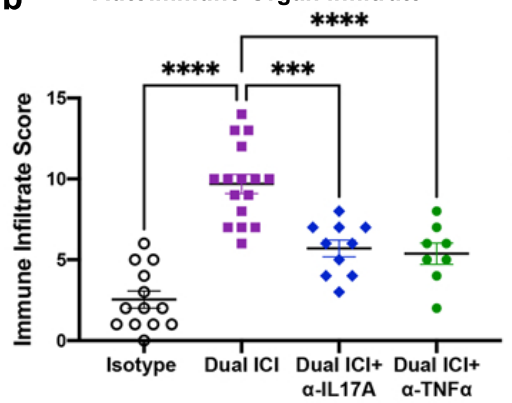

Isotype

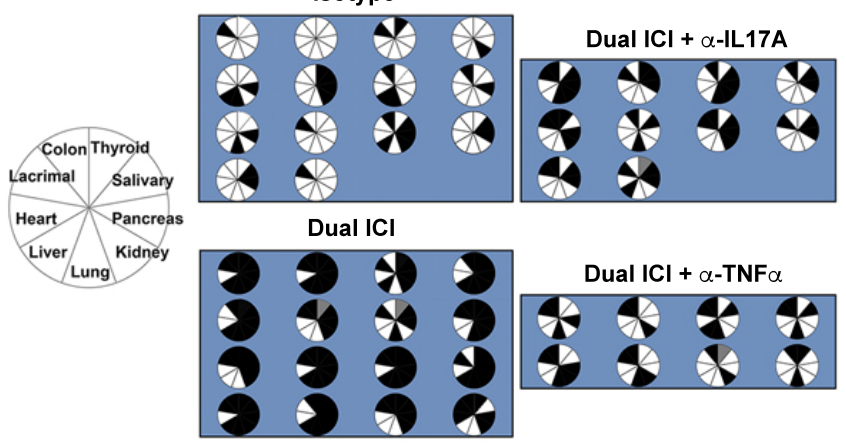

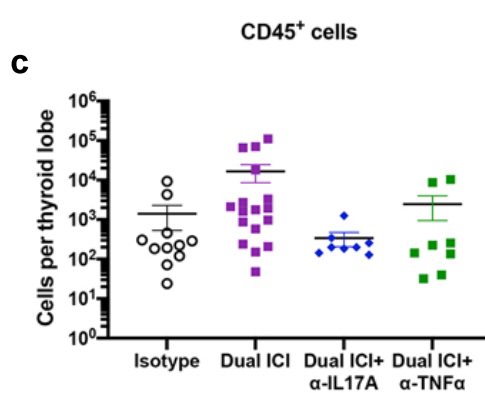

CD11c $^{+}$CD11b ${ }^{\text {low }}$ cells

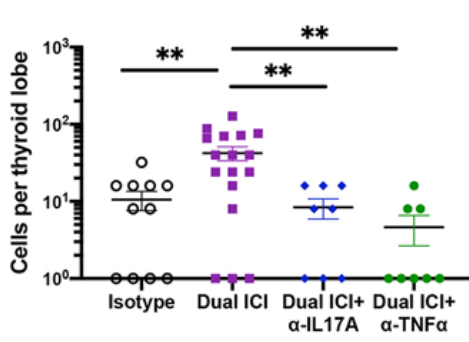

NKp46 ${ }^{+}$CD3- $3^{-}$K cells

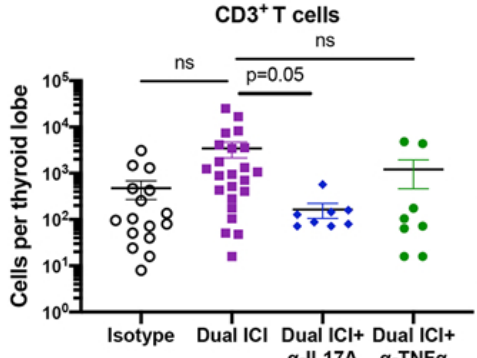

$\alpha$-IL17A $\alpha$-TNF $\alpha$ CD11c $^{+}$CD11 $b^{+}$cells

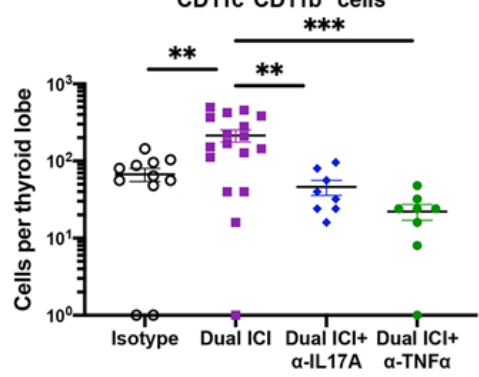

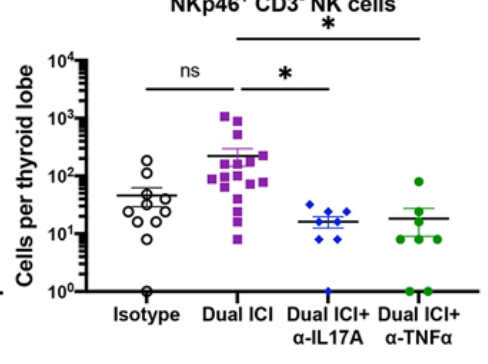

$\mathrm{B}^{2} 20^{+} \mathrm{B}$ cells

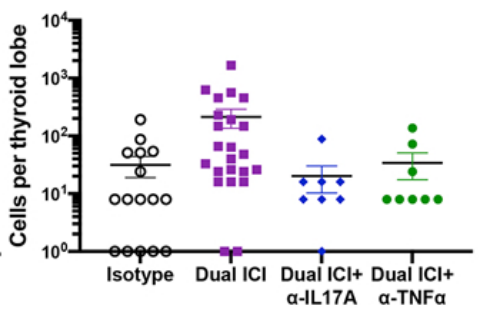

d

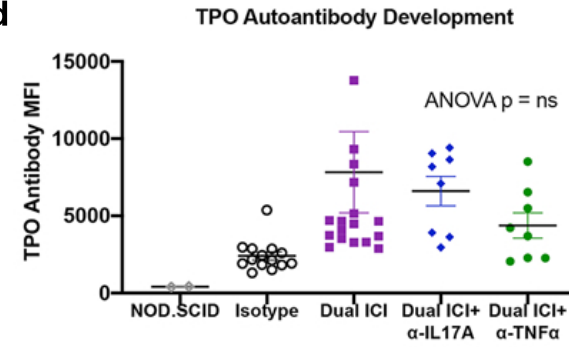

Tg Autoantibody Development

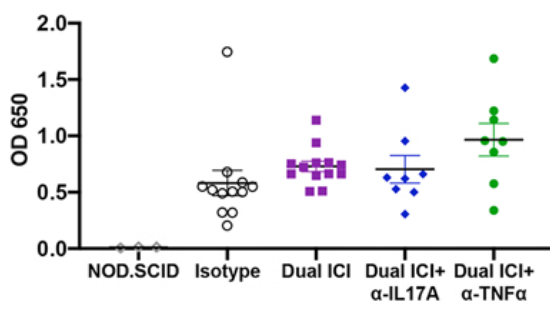

e

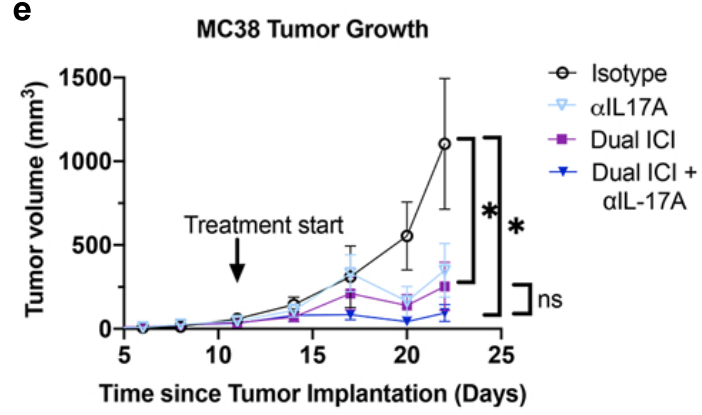

Figure 5. Neutralizing IL-17A or TNF $\alpha$ antibody reduces anti-PD-1 + anti-CTLA-4 (Dual ICI)associated autoimmune infiltrates.

a, Schematic of treatment regimen. $\mathbf{b}$, Autoimmune organ infiltrate score after 4 weeks of $\mathrm{ICl}$ treatment in isotype ( $n=14)$, Dual ICI ( $n=16)$, Dual ICI + neutralizing IL-17A antibody ( $\alpha \mathrm{IL} 17 \mathrm{~A}, n=7)$, and Dual ICI + neutralizing TNF $\alpha$ antibody ( $\alpha$ TNF $\alpha, n=8$ ). Pie charts (right) showing tissues with immune infiltration after 4 weeks of $\mathrm{ICl}$ treatment. Each pie represents one animal; black $=$ immune infiltrate, white $=$ no infiltrate, gray $=$ no data. $\mathbf{c}$, Intrathyroidal immune cell frequency among groups [isotype, $n=11$, except $n=16$ for $\left.\mathrm{CD}^{+}\right)$, Dual ICI $\left(n=17\right.$, except $n=23$ for $\left.\mathrm{CD}^{+}\right)$, Dual ICI $+\alpha \operatorname{LL} 17 \mathrm{~A}(n=8)$, Dual ICI $\left.+\alpha \mathrm{TNF} \alpha(n=8)\right]$. d, Antithyroid autoantibodies in NOD mice after 4 weeks of treatment with isotype ( $n=14$ for anti-TPO, $n=12$ for anti-Tg), Dual ICI ( $n=18$ for anti-TPO, $n=13$ for anti-Tg), Dual ICI $+\alpha \operatorname{IL} 17 \mathrm{~A}(n=8)$, Dual ICI $+\alpha$ TNFa $(n=8)$. e, Growth of MC38 tumors in B6 mice treated with anti-PD-1 + anti-CTLA-4 (Dual ICI) or isotype control, as well as a neutralizing IL-17A ( $\alpha$ IL17A) or relevant isotype, time ( $n=6$ each group). Data are mean \pm SEM shown. ${ }^{* *} p<0.01,{ }^{* * *} p<0.001,{ }^{* * * *} p<0.0001$. Brown-Forsythe ANOVA, assuming unequal s.d., followed by Dunnett's multiple comparisons test as indicated (b-d), or ANOVA for tumor volume at day 22, followed by Dunnett's multiple comparisons test (e). 
a

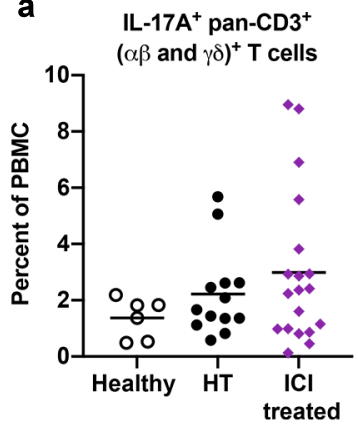

IL-17A ${ }^{+} \mathrm{CD4}^{+} \mathrm{T}$ cells

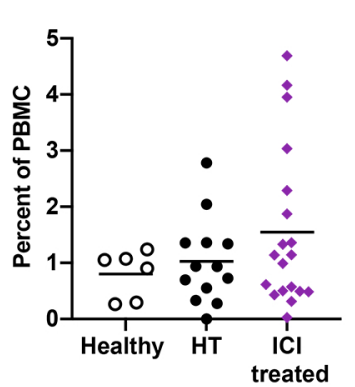

IL-17A ${ }^{+} \gamma \delta$ T cells

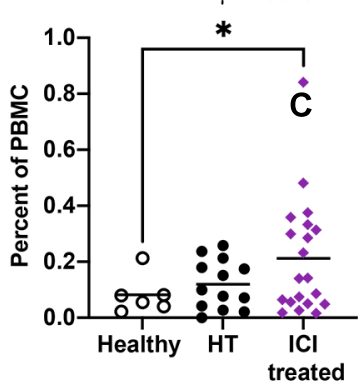

b ICI-associated Thyroid IrAE

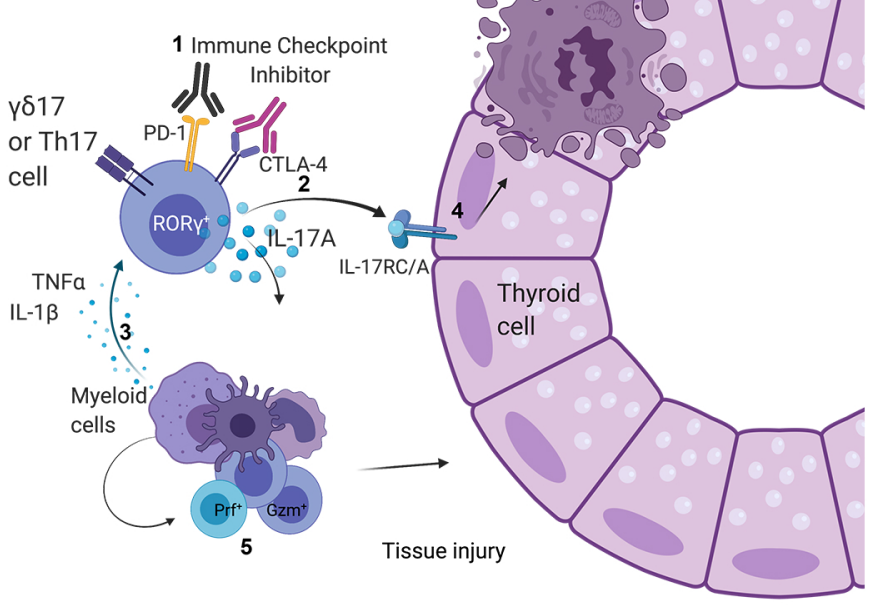

IL-17A axis blockade

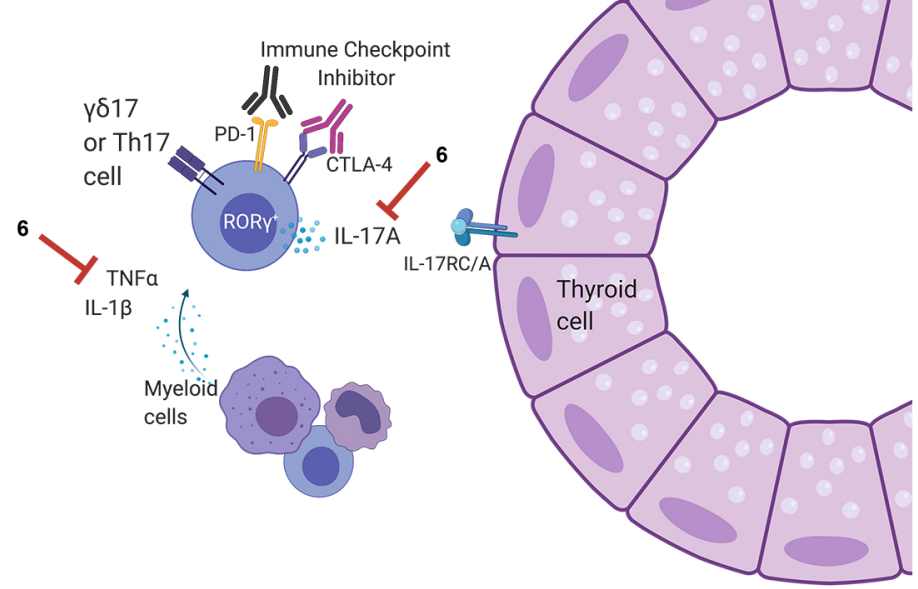

Figure 6. $\gamma \delta \mathrm{T} 17$ and Th17 cells in ICI-treated cancer patients.

a, Relative frequency of IL-17A ${ }^{+}$pan-CD3 ${ }^{+}$, Th17, and $\gamma \delta \mathrm{T} 17$ cells in peripheral blood of controls $(n=6)$, patients with Hashimoto's thyroiditis (HT, $n=13)$, or patients with cancer treated with ICI $(n=20)$. Mean shown. *p<0.05. Brown-Forsythe ANOVA, assuming unequal s.d., followed by Dunnett's multiple comparisons test. b. Proposed mechanism of Thyroid IrAE. (1) ROR $\gamma \mathrm{t}^{+}$Th17 and $\gamma \delta \mathrm{T} 17$ cells may be activated by anti-PD-1 and/or anti-CTLA-4 inhibitor binding to (2) release IL-17A. (3) In addition, myeloid cells within the thyroid may contribute to Th17 and $\gamma \delta \mathrm{T} 17$ activation and recruitment by production inflammatory cytokines IL-1 $\beta$ and TNF $\alpha$ and chemokines. IL-17A within the thyroid microenvironment may affect thyroid follicular cell death via (4) induction of apoptosis or indirectly by (5) facilitating the accumulation of cytotoxic effector cells. (6) Strategies to reduce IrAE target the IL-17A axis. 


\section{Supplementary Files}

This is a list of supplementary files associated with this preprint. Click to download.

- SupplementalMethods.pdf

- Extendeddatafiles.pdf

- ExtDataTable1TopGeneExpressionscRNAseq.xls

- ExtDataTable2CellChat.xlsx 